\title{
A Verticillium dahliae Extracellular Cutinase Modulates Plant Immune Responses
}

\author{
Yue-Jing Gui, ${ }^{1}$ Wen-Qi Zhang, ${ }^{1}$ Dan-Dan Zhang, ${ }^{1}$ Lei Zhou ${ }^{1}$, Dylan P. G. Short, ${ }^{2}$ Jie Wang, ${ }^{1}$ \\ Xue-Feng Ma, ${ }^{1}$ Ting-Gang Li, ${ }^{1}$ Zhi-Qiang Kong, ${ }^{1}$ Bao-Li Wang, ${ }^{1}$ Dan Wang, ${ }^{1}$ Nan-Yang Li, ${ }^{1}$ \\ Krishna V. Subbarao, ${ }^{2,+}$ Jie-Yin Chen, ${ }^{1,+}$ and Xiao-Feng Dai ${ }^{1,+}$ \\ ${ }^{1}$ Laboratory of Cotton Disease, Institute of Food Science and Technology, Chinese Academy of Agricultural Sciences, Beijing, \\ 100193, China; and ${ }^{2}$ Department of Plant Pathology, University of California, Davis, U.S.A.
}

Accepted 4 October 2017.

Cutinases have been implicated as important enzymes during the process of fungal infection of aerial plant organs. The function of cutinases in the disease cycle of fungal pathogens that invade plants through the roots has been less studied. Here, functional analysis of 13 cutinase (carbohydrate esterase family 5 domain-containing) genes ( $V d C U T s$ ) in the highly virulent vascular wilt pathogen Verticillium dahliae Vd991 was performed. Significant sequence divergence in cutinase family members was observed in the genome of $V$. dahliae $\mathrm{Vd}$ 991. Functional analyses demonstrated that only VdCUT11, as purified protein, induced cell death and triggered defense responses in Nicotiana benthamiana, cotton, and tomato plants. Virus-induced gene silencing showed that VdCUT11 induces plant defense responses in Nicotiana benthamania in a BAK1 and SOBIR-dependent manner. Furthermore, coinfiltration assays revealed that the carbohydrate-binding module family 1 protein (VdCBM1) suppressed VdCUT11-induced cell death and other defense responses in $N$. benthamiana. Targeted deletion of VdCUT11 in $V$. dahliae significantly compromised virulence on cotton plants. The cutinase VdCUT11 is an important secreted enzyme and virulence factor that elicits plant defense responses in the absence of VdCBM1.

Plants rely mostly on innate immune responses for defense against potential pathogenic microbes in the environment (Zipfel 2014). At least two innate defense systems operate in plant cells to limit the ingress of infectious microbes. Recognition of pathogens occurs via pattern-recognition receptors (PRRs) and intracellular immune receptors result in PRR-triggered immunity (PTI) and effector-triggered immunity (ETI) responses, respectively (Jones and Dangl 2006; Zipfel 2008). Recognition of a pathogen generally leads to reactive oxygen species (ROS) bursts, callose depositions, and induction of defense-related genes (Boller and Felix 2009; Zipfel 2008). Successful pathogens can overcome these immunity responses via secreted effectors

Yue-Jing Gui, Wen-Qi Zhang, and Dan-Dan Zhang contributed equally to this work.

${ }^{\dagger}$ Corresponding authors: Xiao-Feng Dai;

E-mail: daixiaofeng_caas@126.com,

Jie-Yin Chen; E-mail: chenjieyin@caas.cn, and

Krishna V. Subbarao; E-mail: kvsubbarao@ucdavis.edu

*The $\boldsymbol{e}$-Xtra logo stands for "electronic extra" and indicates that five supplementary figures and five supplementary tables are published online.

(c) 2018 The American Phytopathological Society that suppress PTI or ETI responses (Jones and Dangl 2006; Zipfel 2008). Ongoing coevolution of pathogens and plants results from the selection for novel pathogen genes that overcome plant defenses and new plant genotypes that sustain immunity (Thomma et al. 2011).

Plants activate the first line of immune responses through recognition of pathogen or damage-associated molecular patterns (PAMPs or DAMPs) (Jones and Dangl 2006; Thomma et al. 2011). PAMPs are highly conserved molecules present in microbes but absent in host tissues (Medzhitov and Janeway 1997) and many proteinaceous PAMPs have been characterized in the past ten years (Wu and Zhou 2013). Plants can also sense selfmolecules (DAMPs) that are available for recognition only after host cell or tissue damage has occurred (Zipfel 2014). Wellknown examples of DAMPs include oligogalacturonides (OGs) that are released during the hydrolysis of homogalacturonan (the major component of pectin) by endo-polygalacturonases (De Lorenzo et al. 2001), the 18-amino acid (aa) peptide systemin, derived from the C-terminus of a 200-residue protein precursor in tomato (Schilmiller and Howe 2005), and the 23-aa plant elicitor peptides (Peps) derived from a 92-aa protein precursor (Huffaker et al. 2006). It is now clear that receptor-like kinases (RLKs) and receptor-like proteins (RLPs) are key PRRs for PAMPs or DAMPs associated with the detection of pathogen invasion (Thomma et al. 2011; Wu and Zhou 2013; Zipfel 2008). Currently, a limited number of PRRs involved in the recognition of DAMPs have been identified and all of them are RLKs (Choi and Klessig 2016; De Lorenzo et al. 2011; Wu and Zhou 2013; Zipfel 2008). Examples include the cell wall-associated kinase WAK1, which is a receptor of OGs, and Peps receptor $1 / 2$ (PEPR1/2) (Brutus et al. 2010; Yamaguchi et al. 2006, 2010). Additional immune receptor complexes involved in DAMPtriggered immunity include the brassinosteroid insensitive 1 (BRI1)-associated receptor kinase 1 (BAK1) and the receptorlike cytoplasmic kinases botrytis-induced kinase 1 (BIK1) and PBS1-like 1 (PBL1) (Liu et al. 2013; Postel et al. 2010).

The plant cell wall provides the first barrier against pathogen invasion. Numerous pathogens secrete plant cell wall-degrading enzymes (PCWDEs) to depolymerize the polysaccharide components of the plant cell wall (Kubicek et al. 2014). PCWDEs have received special attention because of their importance in fungal penetration and successful infection of their hosts (Zhao et al. 2014). Comparative genomic analyses have revealed that fungi can produce structurally and functionally varied carbohydrateactive enzymes (CAZymes) and that diversification and expansion of PCWDEs is significantly associated with plant pathogens in particular (Kubicek et al. 2014; Zhao et al. 2014). The cuticle is a protectant film that covers the epidermal cell wall and protects 
the plant against environmental stresses, including pathogen invasion (Chen et al. 2013). Many phytopathogens have evolved the ability to degrade the cuticle by secreting cutinases (Chen et al. 2013; Kolattukudy 1985). In general, cutinases can hydrolyze natural polyesters that act as permeability layers (e.g., cutin and suberin) as well as insoluble triacylglycerols and low-molecular weight soluble esters (Carvalho et al. 1999; Chen et al. 2013; Järvinen et al. 2009; Kontkanen et al. 2009). Fungal enzymes able to degrade cutin may also degrade suberin, e.g., CcCUT1 (Kontkanen et al. 2009; Järvinen et al. 2009). Cutinases are serine esterases that belong to the $\alpha / \beta$ hydrolase superfamily, which possess a classical Ser-His-Asp catalytic triad and two conserved motifs of GYSQG and C-x(3)-D-x-[IV]-C-x-G-[GST]-x(2)[LIVM]-x(2,3)-H (Chen et al. 2013; Egmond and de Vlieg 2000; Martinez et al. 1992).

Depending on the pathogen, cutinases may or may not be strictly required for pathogenicity. Some members of the Fusarium solani species complex (e.g., Fusarium solani f. sp. pisi) secrete cutinases during the penetration of pea stems, and a cutinasedisrupted mutant showed a significant decrease in virulence on pea (Rogers et al. 1994). In other phytopathogens, deletion of cutinase coding genes reduces virulence or eliminates pathogenicity on host plants (Davies et al. 2000; Dickman and Patil 1986; Li et al. 2003; Liu et al. 2016; Skamnioti and Gurr 2007; Wang et al. 2017; Zhang et al. 2005). However, in other studies, cutinase knockout mutants did not lose pathogenicity (Chasan 1992; Stahl and Schafer 1992; Sweigard et al. 1992).

To date, several studies have demonstrated that cutinases are also associated with the elicitation of host-derived signals, fungal spore attachment, and carbon acquisition during saprophytic growth (Cheung et al. 2009; Deising et al. 1992; Järvinen et al. 2009; Kolattukudy 2003; Kolattukudy et al. 1995; Köller et al. 1991; Skamnioti and Gurr 2007). A cutinase was shown to be required for spore adhesion in the rust fungus Uromyces viciae-fabae (Deising et al. 1992), and a cutinase was proposed to be involved in spore adhesion in Colletotrichum graminicola (Pascholati et al. 1993). Cutinases may also play a role in inducing defense responses (Parker and Köller 1998; Zhang et al. 2014), such as in the pathogen Sclerotinia sclerotiorum, in which SsCut acts as a PAMP to induce defense responses in several host plants (Zhang et al. 2014). Degradation of plant cuticles by cutinases secreted by fungal pathogens may lead to the release of DAMPs (De Lorenzo et al. 2011). For example, free cutin monomers resulting from the degradation of the cuticle can play an important role in plant resistance (Chassot and Metraux 2005; Schweizer et al. 1996a) by acting as DAMPs (Kauss et al. 1999; Kim et al. 2008; Schweizer et al. 1996a), although the one or more receptors remains unknown. Interestingly, experiments with plant cutin mutants showed immunity to the necrotrophic fungus Botrytis cinerea (Bessire et al. 2007; Chassot et al. 2007).

Verticillium dahliae is a soil-borne vascular wilt fungal plant pathogen that causes a widespread and destructive wilt disease on many hosts, including economically important crops (Klosterman et al. 2009). The reference genome of the hemibiotrophic pathogen Verticillium dahliae Vd.Ls17 is replete with pectinolytic enzymes that are hypothesized to greatly enhance the capacity of the fungus to breach defense structures and enable colonization of plant xylem vessels (Klosterman et al. 2011) and contribute to its vast host range. Previous studies showed that the expansion of PCWDEs played an important role in adaptation to plant xylem vessels (Chen et al. 2016; Klosterman et al. 2011). Specifically, several PCWDEs are involved in pathogenicity and virulence, including the glucosyltransferase homolog gene VDAG_02071, which is required for full virulence in Nicotiana benthamiana (Klosterman et al. 2011), glycoside hydrolase 12 (GH12) proteins, and polysaccharide lyase family 3 (PL3) (Chen et al. 2016).
Regarding cutinases (family CE5) in $V$. dahliae, comparative analyses revealed that the $V$. dahliae reference genome (strain VdLs.17) encodes at least 14 cutinase members (Klosterman et al. 2011), suggesting that cutinases play an important role during pathogenesis. However, unlike the hemibiotrophic rice blast fungus Magnaporthe oryzae, the reference genome of which encodes 19 cutinases putatively involved in-cuticle degradation of leaves (Zhao et al. 2014), V. dahliae is a soil-borne pathogen that infects the plant xylem through root tissue that generally does not contain a cuticle. Thus, the function of the large cutinase family in $V$. dahliae is unclear, especially during pathogenesis.

Carbohydrate binding modules (CBM) are noncatalytic protein domains that are generally associated with carbohydrate hydrolases activity in fungi and which can, in some cases, trigger or suppress plant defense responses (Gaulin et al. 2006; Gui et al. 2017). In V. dahliae Vd991 specifically, a protein with a single CBM domain (VdCBM1, homologous to Vd.Ls17 VDAG_00170) suppressed the recognition of key virulenceassociated cellulases in $N$. benthamiana (Gui et al. 2017).

In the current study, the cutinase family of V. dahliae Vd991, isolated from the host Gossypium hirsutum (cotton), was investigated. The main objectives were to i) identify members of the cutinase gene family in V. dahliae Vd991, ii) determine which cutinase family members trigger an immune response in $N$. bethamiana, using a transient expression assay, iii) elucidate the coreceptor associated with PRRs involved in triggering immunity in the model plant $N$. benthamiana, iv) study the activity of an effector of $V$. dahliae in suppressing immunity triggered by the cutinase family, and v) investigate the function of a key cutinase during pathogenesis of $G$. hirsutum roots.

\section{RESULTS}

\section{Identification of the cutinase family in the genome} of Verticillium dahliae Vd991 pathogenic to cotton.

Thirteen cutinase domain-containing proteins were identified in the proteome predicted from the $V$. dahliae Vd991 genome sequence (Chen et al. 2017), using HMMER with the hidden Markov model (HMM) file of the CE5 domain (PF01083) and BLASTP searches of the CAZymes database. The 13 proteins were designated VdCUT1 through VdCUT13; all contained the CE5 domain, while only VdCUT11 contained a CBM1 domain (Supplementary Table S1). Of the cutinase family members in Vd991, all except VdCUT2 were predicted as secreted proteins, due to the presence of a typical signal peptide and the absence of a transmembrane domain (Supplementary Table S2). All except VdCUT2 were small cysteine-rich proteins $(<400$ aa, more than four cysteine residues). BLAST analyses revealed high aa sequence identity, of all cutinase family members, between Vd991 and VdLs.17 (Supplementary Table S3).

An aa sequence alignment including all cutinases from V. dahliae Vd991 and Vd.Ls17 along with seven known cutinases from other plant pathogens was generated. Eleven Vd991 cutinases had highly conserved P-x-[STA]-x-[LIV]-[IVT]-X[GS]-G-Y-S- $[Q L]-G$ (the enzymatic activity site $S$ is underscored) and C-x(3)-D-x-[IV]-C-x-G-[GST]-x(2)-[LIVM]-x(2,3)(enzymatic activity sites $\mathrm{D}$ and $\mathrm{H}$ are underscored) motifs similar to cutinases from other phytopathogens. All Vd991 cutinases had these motifs except for VdCUT2 and VdCUT3, in which the domains were truncated or missing from the C-terminus (Fig. 1A). Phylogenetic analysis showed that $V$. dahliae cutinase family members were sorted into three distinct groups comprising five, three, and five cutinases each (Fig. 1B). The known cutinases also were distributed into three branches (Fig. 1B), clustered according to biological activity. For example, the reference proteins known to be involved in virulence, triggering defense responses, or spore adhesion clustered into 


A MgCUT2
VdCUT5
MgCUT1
VdCUT10
VdCUT12
VdCUT9
VdCUT2
VdCUT3
VdCUT4
BgCUT1
MfCUT1
SsCut1
Pbc1
VdCUT1
VdCUT11
VdCUT8
VdCUT13
VdCUT6
VdCUT7
CICUT7
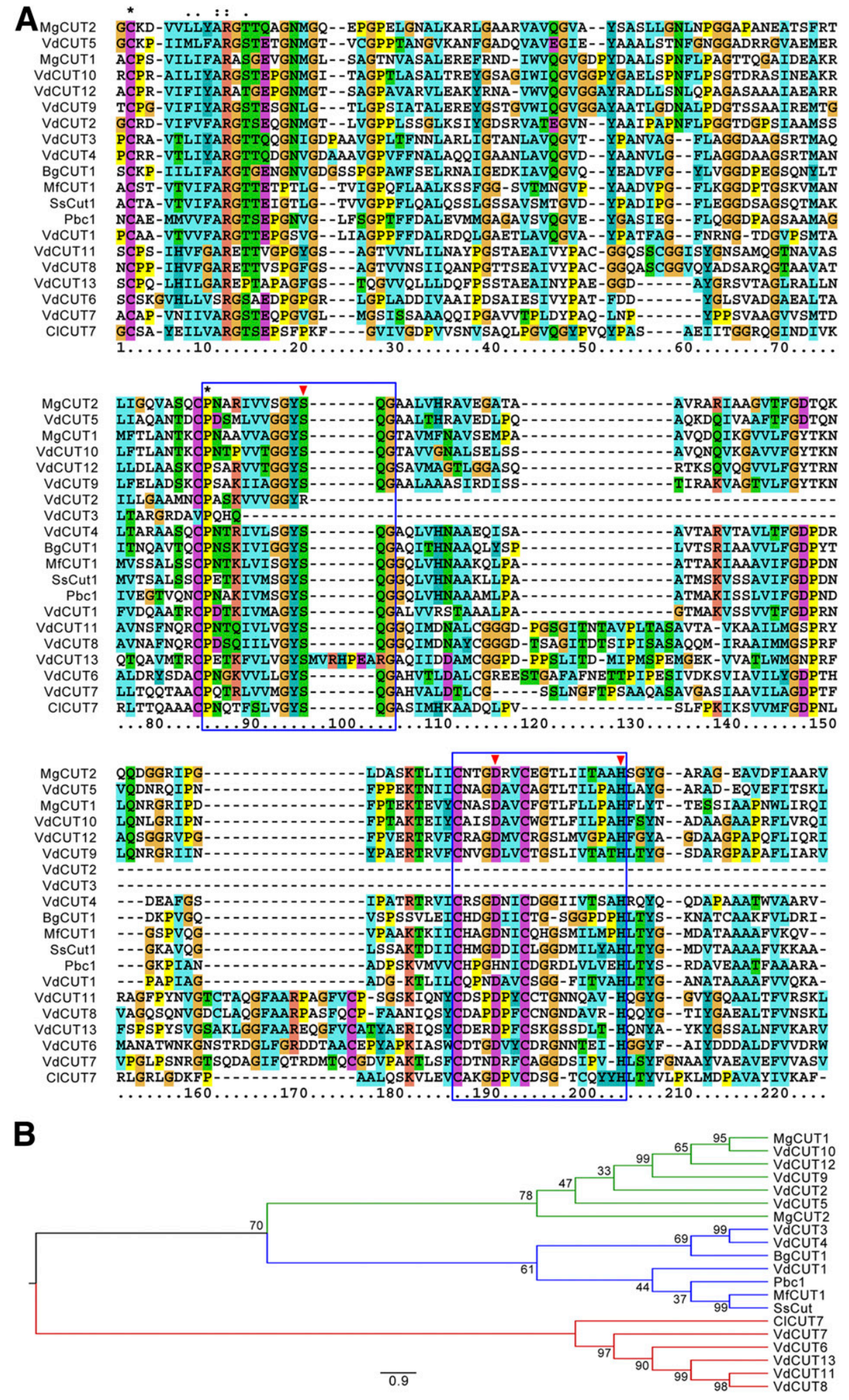

Fig. 1. Bioinformatics of the cutinase family in Verticillium dahliae. A, Sequence alignment of $V$. dahliae cutinase family proteins with known cutinases from other fungi. The CE5 domain sequences were extracted from the cutinase family (VdCUT1 to VdCUT13) according to HMMer prediction results, and sequence alignments were performed with ClustalX 1.83; asterisks represent conserved residues. Two conserved motifs in the cutinase family, P-x-[STA]- $\mathrm{x}-$ [LIV]-[IVT]-x-[GS]-G-Y-S-[QL]-G and C-x(3)-D-x-[IV]-C-x-G-[GST]-x(2)-[LIVM]-x(2,3)-H are highlighted in blue boxes; red triangles indicate the enzymatic activity sites. The accession numbers of known cutinases are MgCUT1 (EHA46959.1), MgCUT2 (EHA51400.1), SsCut1 (EDN91800.1), BgCUT1 (AF326784.1), MfCUT1 (AF305598.1), Pbc1 (AJ009953.1), and ClCUT7 (Liu et al. 2016). B, Phylogenetic relationships between V. dahliae cutinase family members and cutinases from other fungi. The phylogeny was constructed by Mega 6.0, using maximum-likelihood (parameters: 1,000 bootstraps, JonesTaylor-Thornton model). Branch colors indicate the three groups of cutinase family members in V. dahliae. 
different groups. This result suggested that the functions of the cutinase family members are significantly divergent in $V$. dahliae Vd991.

\section{Verticillium dahliae Vd991 secreted cutinase VdCUT11 induces cell death in $N$. benthamiana.}

To determine the cell death-inducing activities of cutinase family members in $V$. dahliae $\mathrm{Vd} 991$, the full-length coding sequence of each cutinase was separately inserted into the Potato virus X (PVX) vector pGR107 and was transformed into Agrobacterium tumefaciens GV3101 for transient expression in 4week-old $N$. benthamiana plants. Independent transient expression of $13 \mathrm{~V}$. dahliae cutinases in $N$. benthamiana resulted in leaf tissue collapse at 3 to 4 days with VdCUT11, followed by obvious cell death or necrosis at 6 days, exclusively following agroinfiltration of VdCUT11 (Fig. 2A). Immunoblot analysis of the total protein extract from the agroinfiltrated leaf area confirmed effective protein translation of all cutinase family members (Fig. 2B). The yeast signal trap assay showed that the signal peptide of VdCUT11 had the ability to mediate the secretion of invertase after fusing with the invertase gene (which lacks a signal peptide in the plasmid pSUC2) in yeast and successfully conferred the ability of yeast strain YTK12 to grow on the medium with raffinose as the sole carbon source and grow normally (Fig. 2C). Based on these observations, the signal peptide of VdCUT11 is sufficient for the secretion of invertase, providing evidence that VdCUT11 is a secreted protein in V. dahliae.

\section{The enzymatic activity of VdCUT11 is required}

\section{for cell death-inducing activity in $N$. benthamiana.}

Three conserved residues have been identified as essential for catalyzing the degradation of the plant cuticle by cutinases (Chen et al. 2013). These three catalytic residues were absolutely conserved in VdCUT11 (Ser-113 in the first motif, Asp-198 and His-210 in the second motif) (Figs. 1A and 3A). To assess the relationship between the enzymatic activity and cell deathinducing activity of VdCUT11, we constructed a site-directed mutant VdCUT11 ${ }^{\mathrm{SM}}$ that contained alanine (Ala) residues substituted at each of the three enzymatic sites (Fig. 3A). Transient expression of VdCUT11 ${ }^{\mathrm{SM}}$ in $N$. benthamiana plants did not result in cell death 6 days after agroinfiltration (Fig. 3B), indicating that the enzymatic activity of VdCUT11 is essential for the cell death-inducing activity in $N$. benthamiana.

To confirm this result, recombinant proteins of the wild-type and site-directed mutagenized genes were purified using the small ubiquitin-related modifier (SUMO) tag (Supplementary Fig. S1). Assays of the 45-kDa recombinant proteins tested against the substrate $p$-nitrophenyl butyrate (pNPB) revealed that wildtype VdCUT11 had hydrolase activity and produced $p$-nitrophenol $(12.74 \pm 0.84 \mathrm{U})$, whereas a nearly complete loss of hydrolase activity $(0.96 \pm 0.32 \mathrm{U})$ was observed for the recombinant
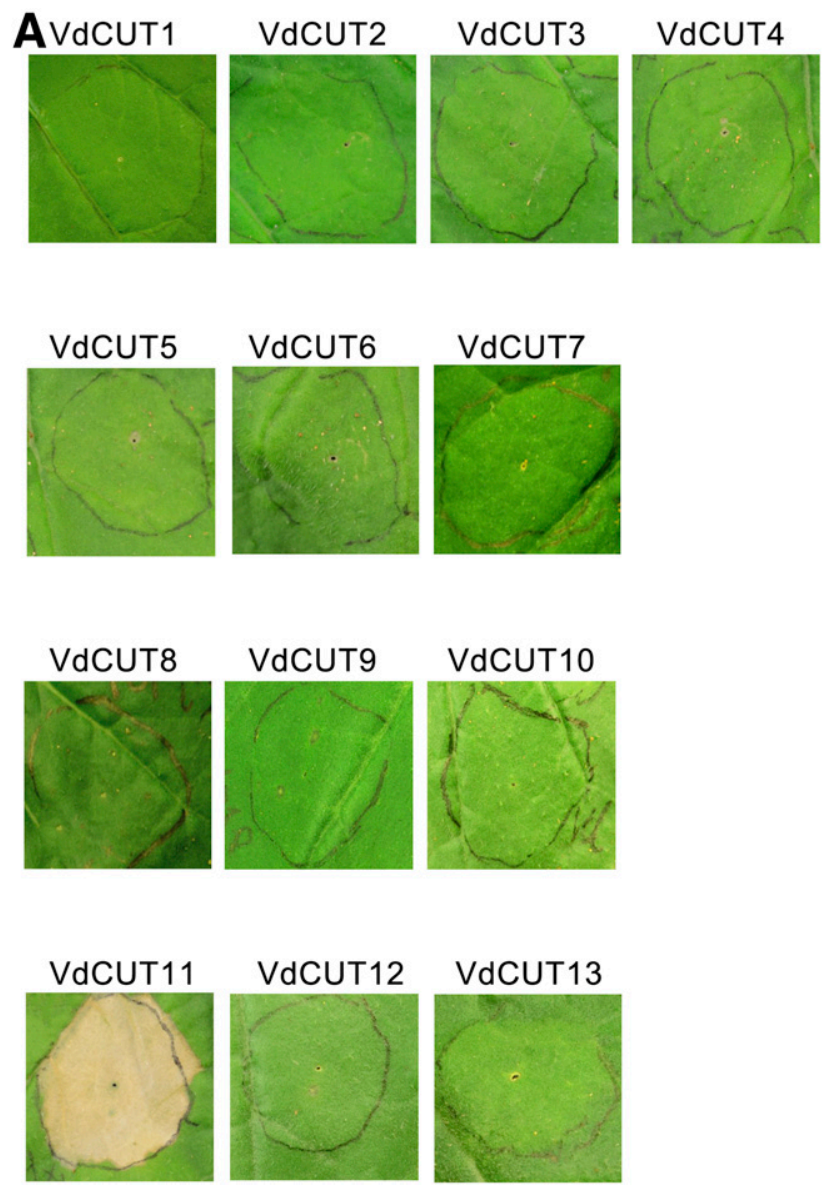

Fig. 2. Identification of cell death-inducing activity of Verticillium dahliae cutinases in Nicotiana benthamiana. A, Cell death induction was detected for $13 \mathrm{~V}$. dahliae cutinase genes (VdCUT1 to VdCUT13) in $N$. benthamiana leaves from 4-week-old plants 6 days after infiltration with Agrobacterium tumefaciens carrying the indicated genes. B, Immunoblotting analysis of transiently expressed cutinase genes fused to the FLAG tag in $N$. benthamiana leaves $60 \mathrm{~h}$ after infiltration. Ponceau S-stained Rubisco protein is shown as a total protein loading control. C, Validation of the secretion function of the signal peptides of VdCUT11 by a yeast signal trap assay. The yeast strain YTK12 could not grow on CMD-W medium without tryptophan. The strain containing the pSUC2 vector grew, based on the function of the Trp operon. Fusion of the functional signal peptide of VdCUT11 in-frame with mature yeast invertase enabled secretion of invertase, resulting in growth on YPRAA medium. The known functional signal peptide Avr1b was used as a positive control. 
protein VdCUT11 ${ }^{\mathrm{SM}}$ (Fig. 3D), suggesting that VdCUT11 has true cutinase functionality. Further, purified VdCUT11 recombinant protein induced cell death in $N$. benthamiana 2 days after infiltration of $5 \mu \mathrm{M}$ protein, much more so than the celldeath symptoms after infiltration of site-directed mutagenized protein VdCUT11 ${ }^{\mathrm{SM}}$ (Fig. 3E). In addition, the infiltration of VdCUT11 and VdCUT11 ${ }^{\text {SM }}$ recombinant proteins into two other hosts showed that VdCUT11, compared with the nonenzymatic mutagenized protein $\mathrm{VdCUT} 11^{\mathrm{SM}}$, induced much more cell death in cotton (Gossypium hirsutum) and tomato (Lycopersicon esculentum) (Fig. 3F and $\mathrm{G}$ ) as in the radicle tissue of the developing cotton seedlings (Fig. $3 \mathrm{H}$ and I). These results suggested that the secreted cutinase VdCUT11, due to its enzymatic activity, lacks host specificity and has the ability to induce cell death in various plants.

\section{VdCUT11 is localized to the apoplast and}

triggers additional defense responses in $N$. benthamiana.

Because VdCUT11 is a secreted protein (Fig. 2C), experiments were conducted to test whether VdCUT11 was localized
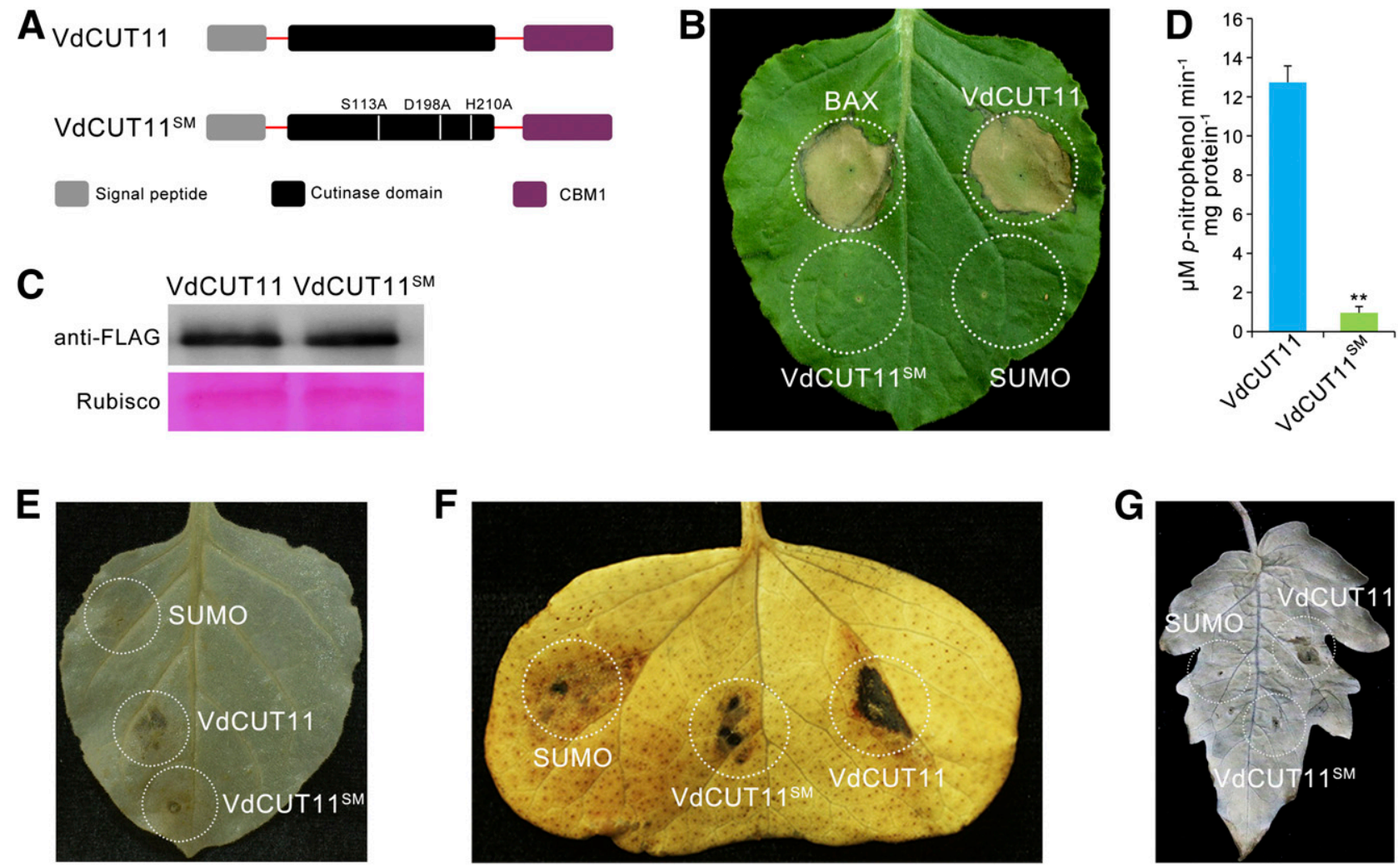

$\mathbf{F}$

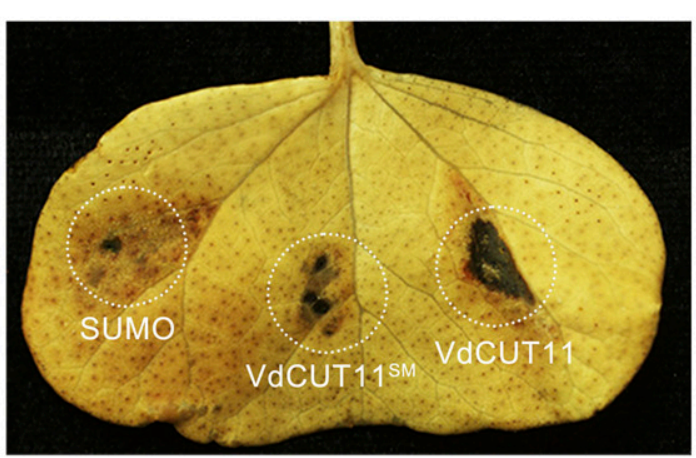

G

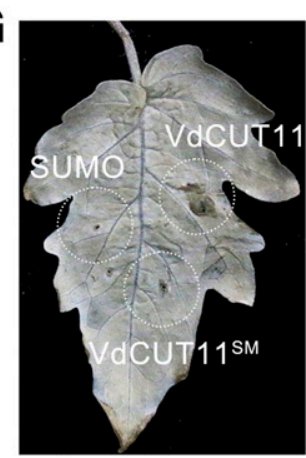

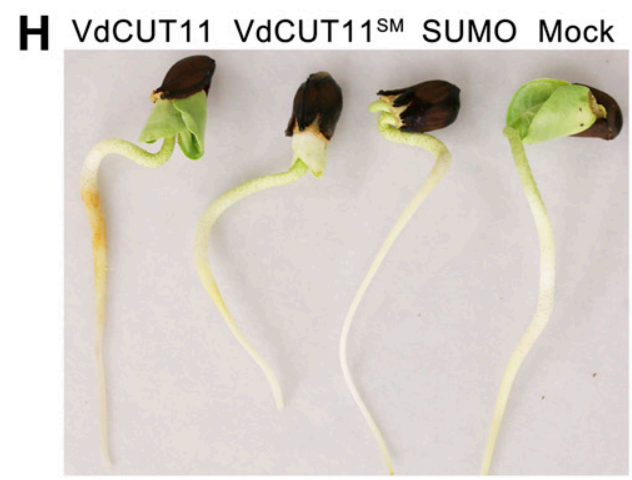

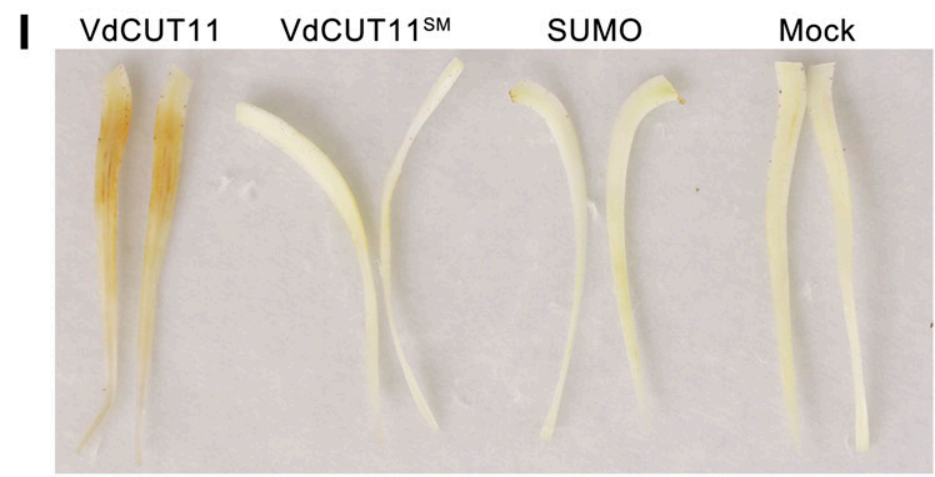

Fig. 3. Enzymatic activity is required for the cell death-inducing activity of VdCUT11. A, Gene structures of wild-type VdCUT11 and locations of sitedirected mutagenesis of three enzymatic activity residues. Gray, black, and purple blocks represent the signal peptide, cutinase domain (CE5), and CBM1 domain, respectively. Three white lines in the black block represent the three mutagenesis sites in the cutinase domain. B, Detection of the cell death-inducing activities of wild-type VdCUT11 and site-directed mutagenesis VdCUT11 ${ }^{\mathrm{SM}}$. Transient expression was assessed in Nicotiana benthamiana leaves from 4-week-old plants 6 days after agroinfiltration, and green fluourescent protein was used as a control. C, Immunoblotting analysis of VdCUT11 and VdCUT11 ${ }^{\text {SM }}$ protein fused to a FLAG tag transiently expressed in N. benthamiana leaves 6 days after agroinfiltration. Ponceau S-stained Rubisco protein is shown as a total protein loading control. D, Detection of the enzymatic activity of wild-type and site-directed mutagenized VdCUT11 toward p-nitrophenyl butyrate. Values represent the averages of three independent measurements with three replicates each. Standard errors are shown. Asterisks (**) indicate significant differences $(P<0.01)$. E to $\mathbf{G}$, Cell-death response in leaves of three other species of plants triggered by $5 \mu \mathrm{M}$ protein VdCUT11 and VdCUT11 ${ }^{\mathrm{SM}}$ recombinant proteins, the small ubiquitin-related modifier (SUMO) tag was used as negative control. Representative leaves are shown of 4-week-old plants of $N$. benthamiana (E), 2-weekold plants of cotton cv. Junmian 1 cotyledon (F), and 2-week-old plants of 'MoneyMaker' tomato (G). H and I, Cell death response in cotton radicle tissue triggered by the root uptake of $5 \mu \mathrm{M}$ protein VdCUT11 and VdCUT11 ${ }^{\mathrm{SM}}$ recombinant proteins, the SUMO tag, and a mock control (dissolution buffer, $25 \mathrm{mM}$ Tris, $500 \mathrm{mM}$ $\mathrm{NaCl}, 0.5 \%$ TritonX -100, $500 \mathrm{mM}$ imidazole, $\mathrm{pH}$ 8.0) were used as positive and negative controls, respectively. Representative radicles of 2-day-old cotton treated with recombinant proteins for $48 \mathrm{~h}(\mathrm{H})$ and the phenotype of vertical sections (I). 
A VdCut11

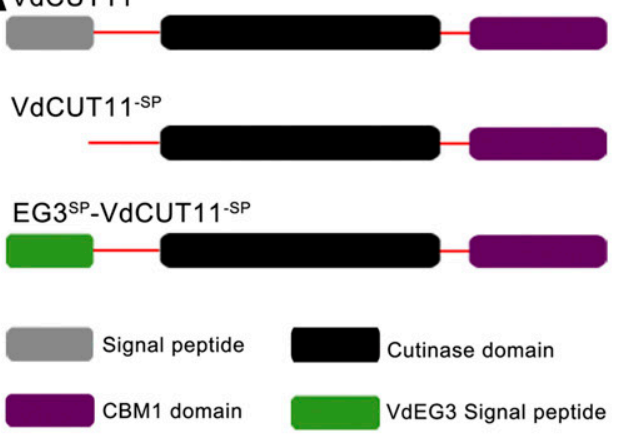

C

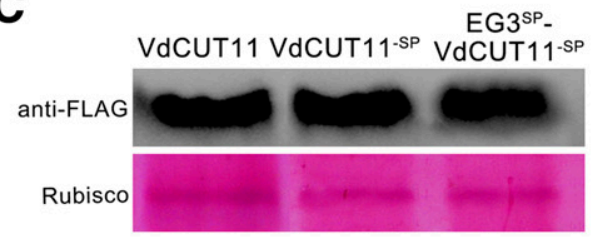

$\mathbf{F}$

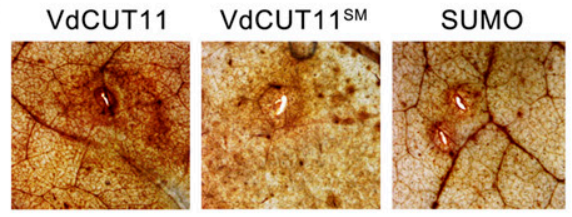

B

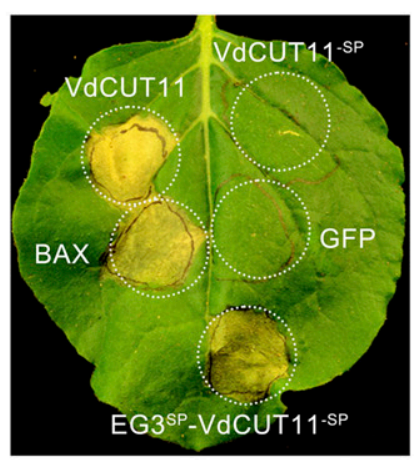

$\mathbf{E}$

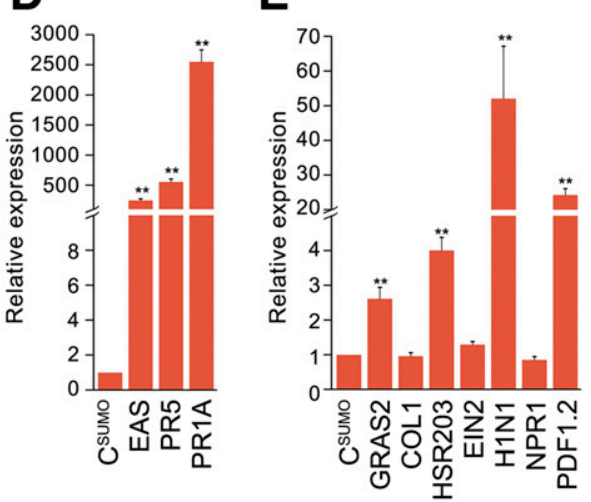

$\mathbf{G}$
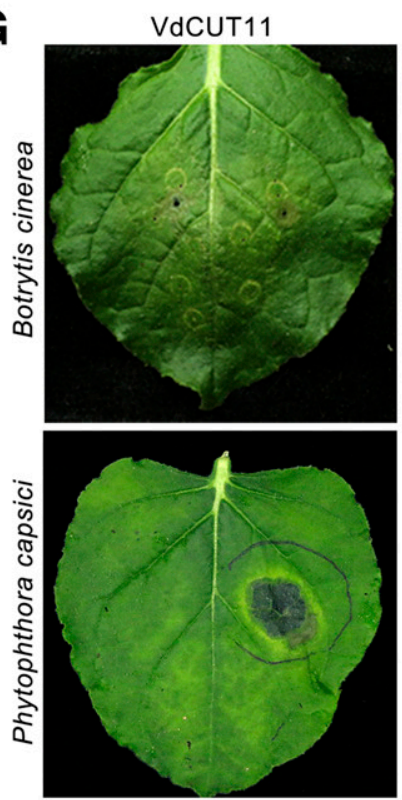
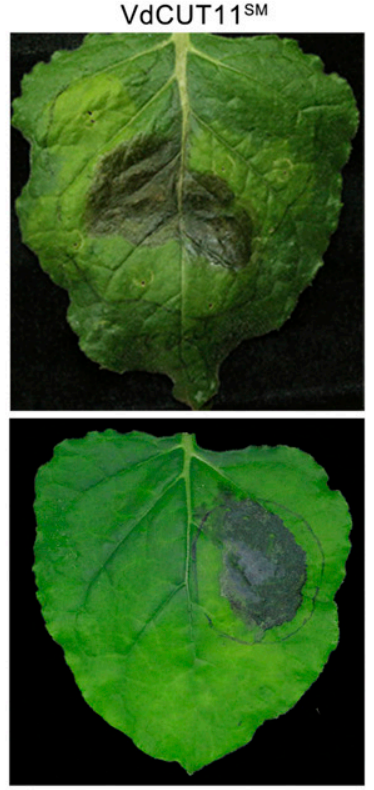
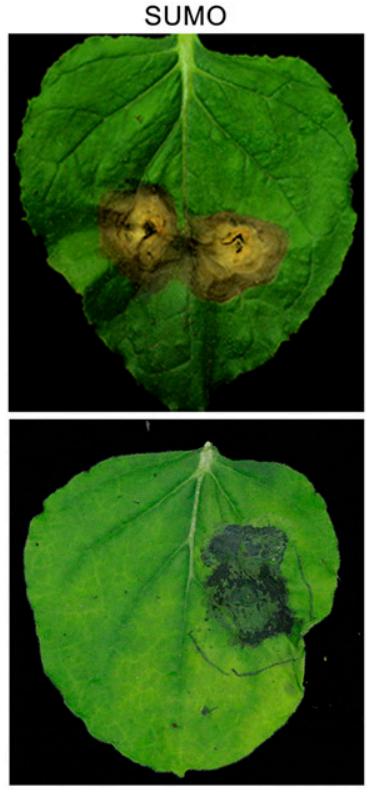

Fig. 4. Identification of defense responses triggered by Verticillium dahliae VdCUT11. A, Gene structures encoding VdCUT11 chimeric proteins with signal peptide deletion or substitution. Blocks in gray, black, and purple represent the signal peptide, cutinase domain (CE5), and CBM1 domain, respectively. A green block represents the VdEG3 signal peptide that has the ability to secret VdEG3 into the apoplast. VdCUT11 $1^{\text {-SP }}$, signal peptide deletion mutant; EG3 ${ }^{\mathrm{SP}}$-VdCUT1 $1^{\text {-SP }}$, a chimeric protein in which the VdEG3 signal peptide has been substituted in place of the native signal peptide of VdCUT11. B, Cell death-induction assay of VdCUT11, VdCUT1 1 ${ }^{-\mathrm{SP}}$, and EG3 ${ }^{\mathrm{SP}}$-VdCUT1 ${ }^{\text {-SP }}$ in Nicotiana benthamiana 4-week-old plants 6 days after agroinfiltration. BAX (Bcl-2-associated X) and green fluorescent protein (GFP) were used as positive and negative controls, respectively. C, Immunoblotting analysis of proteins in $N$. benthamiana leaves transiently expressing VdCUT1 $1^{\text {-SP }}$ and EG3SP-VdCUT1 $1^{\text {-SP }}$; Ponceau S-stained Rubisco protein is shown as a total protein loading control. D and E, Detection of transcripts of defense response genes using quantitative reverse transcription-polymerase chain reaction. The transcripts were detected in 4-week-old $N$. benthamiana leaves $24 \mathrm{~h}$ after infiltration of recombinant VdCUT11 recombinant protein $(400 \mathrm{nM})$. The level of transcripts of defense genes after infiltration of small ubiquitin-related modifier (SUMO)-tagged protein was used as a control $\left(\mathrm{C}^{\mathrm{SUMO}}\right)$. Values represent the averages of three independent measurements with three replicates each. Error bars represent standard errors. F, Reactive oxygen species accumulation was assessed in $N$. benthamiana leaves from 4-week-old plants $12 \mathrm{~h}$ after infiltration with $400 \mathrm{nM}$ VdCUT11 and VdCUT11 ${ }^{\text {SM }}$ purified recombinant protein; SUMO tag was used as negative control. Leaves were stained with 3'3-diaminobenzidine. G, Defense response induced by pretreatment with recombinant VdCUT11 and VdCUT11 ${ }^{\mathrm{SM}}$ recombinant proteins. N. benthamiana leaves from a 4-week-old plant were pretreated with $100 \mathrm{nM}$ of the indicated recombinant protein and were inoculated $12 \mathrm{~h}$ later with $5.0 \mu \mathrm{l}$ of $2 \times 10^{6}$-conidia/ml solutions of Botrytis cinerea and Phytophthora capsici. Lesion symptoms were observed at 2 days postinoculation. 
to the plant apoplast. Transient expression of the N-terminal signal peptide deletion mutant (VdCUT11 ${ }^{\text {-SP }}$ ) (Fig. 4A) did not trigger cell death in $N$. benthamiana 6 days after agroinfiltration (Fig. 4B), although VdCUT11 $1^{\text {-SP }}$ was successfully detected in planta by immunoblot analysis (Fig. 4C). Transient expression of the chimeric sequence EG3 ${ }^{\mathrm{SP}}$-VdCUT1 $1^{\text {-SP }}$, in which the missing signal peptide of $\mathrm{VdCUT} 11^{-\mathrm{SP}}$ was complemented with the known apoplast targeting signal peptide from VdEG3 (Gui et al. 2017), recovered the ability to trigger cell death in $N$. benthamiana 6 days after agroinfiltration (Fig. 4B and C). These results suggested that VdCUT11 is specifically localized to the apoplast.

To test whether VdCUT11 triggered an immunity response in $N$. benthamiana, we measured the relative expression of several genes associated with plant defense responses after treatment with recombinant protein. As expected, several defense-related genes were significantly up-regulated in $N$. benthamiana leaves $24 \mathrm{~h}$ after treatment (Fig. 4D and E), especially the transcript levels of 5-epi-aristolochene synthase (EAS), pathogenesisrelated gene 5 (PR5), and PR1a, which were up-regulated more than 100-fold (Fig. 4D). DAB (3'3-diaminobenzidine) staining of $N$. benthamiana leaves showed that VdCUT11 but not VdCUT $11^{\mathrm{SM}}$ had the ability to activate ROS accumulation (Fig. 4F). Furthermore, the development of B.cinerea infection was significantly reduced in $N$. benthamiana leaves pretreated with $400 \mathrm{nM}$ VdCUT11 recombinant protein, but leaves pretreated with VdCUT11 ${ }^{\mathrm{SM}}$ recombinant protein displayed obvious lesion development $48 \mathrm{~h}$ after inoculation with $B$. cinere $a$ and Phytophthora capsici (Fig. 4G). Together, these results strongly suggested that purified VdCUT11 triggered plant defense responses.

\section{BAK1 and SOBIR1 are required}

\section{for VdCUT11-triggered immunity in $N$. benthamiana.}

Based on its localization to the apoplast, (Fig. 2C and Fig. 4B) the immunity triggered by VdCUT11 should depend on surfacelocalized PRRs, either RLKs or RLPs. To further characterize VdCUT11-triggered immunity, virus-induced gene silencing (VIGS) constructs based on recombinant Tobacco rattle virus (TRV) were generated to target $N b B A K 1$ and NbSOBIRl expression in tobacco, two genes that play important roles in the regulation of different ligand-binding receptors (Monaghan and Zipfel 2012; Liebrand et al. 2013; Zhang et al. 2013). Three weeks after viral inoculation to silence $N b B A K 1$, transient expression of VdCUT11 in $N$. benthamiana did not result in cell death after agroinfiltration with VdCUT11 expression constructs. Treatment of $N b B A K 1$-silenced plants with Bcl-2-associated protein $\mathrm{X}(\mathrm{BAX})$ was used as a control, which resulted in cell death induction (Fig. 5A). Similarly, in NbSOBIRl-silenced plants, the cell death-induction was completely absent after agroinfiltration with VdCUT11 expression constructs (Fig. $5 \mathrm{~A})$. Immunoblotting confirmed that VdCUT11 was successfully expressed in $N$. benthamiana inoculated with TRV::BAK, TRV::SOBIR1, or TRV::GFP (Fig. 5B). Quantitative reverse transcription-polymerase chain reaction (qRT-PCR) analysis confirmed that the expression of $N b B A K 1$ or $N b S O B I R 1$ expression was markedly reduced upon inoculation with the TRV:: $B A K$ or TRV::SOBIR1, with an expression level about $20 \%$ compared with inoculation with TRV::GFP (Fig. 5C and 5D). SOBIR1 appears to be specifically required for the function of receptor complexes containing RLP that the lack an intracellular signaling domain (Liebrand et al. 2014). Therefore, these
A

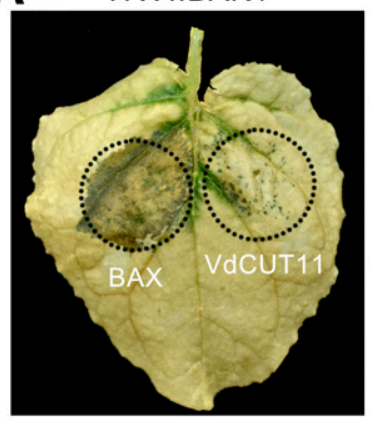

B
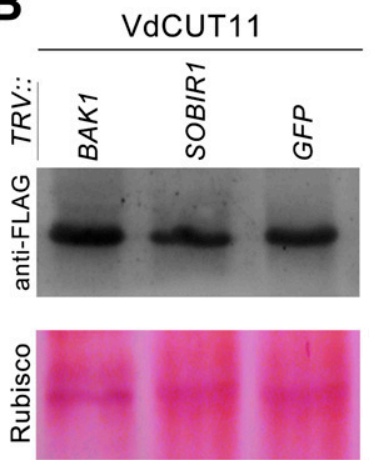

TRV::SOBIR1

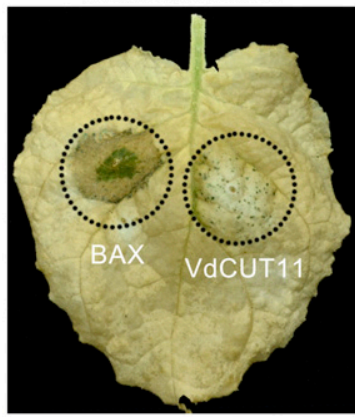

C

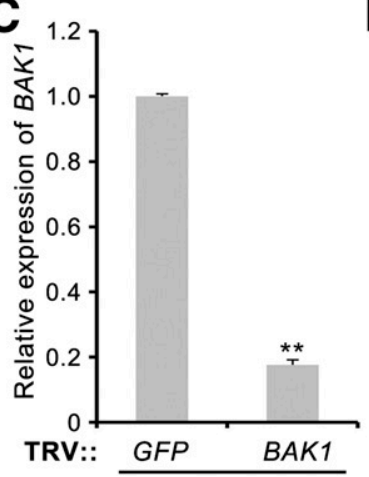

TRV::GFP

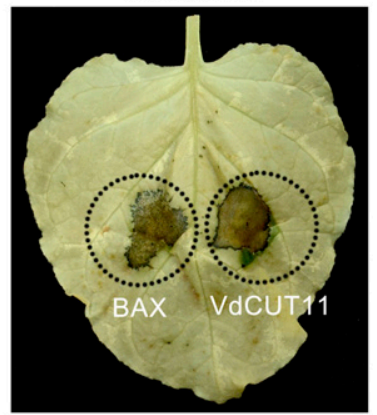

D

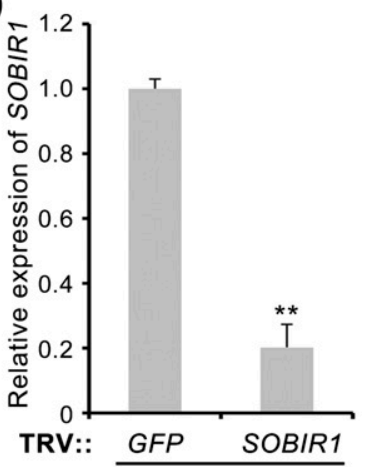

Fig. 5. Analysis of the associations between VdCUT11 and immunity receptors of Nicotiana benthamiana. A, BAK1 and SOBIR1 are required for VdCUT11triggered cell death in $N$. benthamiana. $N$. benthamiana plants were subjected to virus-induced gene silencing by inoculation with Tobacco rattle virus (TRV) constructs (TRV:NbBAK1, TRV::NbSOBIR1, or TRV::GFP). Three weeks after inoculation, VdCUT11 and the BAX (Bcl-2-associated X) positive control were transiently expressed in gene-silenced leaves, and the phenotypes of induced cell death were photographed 6 days later. The experiment was performed three times with six plants for each TRV construct. B, Immunoblotting analysis of VdCUT11 fused to a FLAG tag transiently expressed in gene-silenced (NbBAK1 or NbSOBIR1) $N$. benthamiana leaves 6 days after agroinfiltration. Ponceau S-stained Rubisco protein is shown as a total protein loading control. $\mathbf{C}$ and $\mathbf{D}$, Determination of the silencing efficiency of immunity receptor genes NbBAK1 (C) and NbSOBIR1 (D) by quantitative reverse transcriptionpolymerase chain reaction analysis. EF- $1 \alpha$ was used as an endogenous control. Means and standard errors from three biological replicates are shown. Asterisks $(* *)$ indicate significant differences $(P<0.01)$ 
results suggested that BAK1 and SOBIR1 (a LRR-RLP/SOBIR1/ BAK1 complex) is required for VdCUT11-triggered immunity in N. benthamiana.

\section{VdCBM1 can suppress}

the VdCUT11-triggered defense response.

Although purified VdCUT11 caused cell death and triggered other defense responses in $N$. benthamiana, we hypothesized that the immunity triggered by VdCUT11 might be suppressed by effectors, resulting in successful host infection by $V$. dahliae. The previously characterized plant immunity suppressor $V$. dahliae carbohydrate-binding module 1 (VdCBM1) (Gui et al. 2017) was tested for its activity to moderate VdCUT11 defense responseinducing activity. Interestingly, coexpression of VdCUT11 and VdCBM1 did not result in cell death in $N$. benthamiana leaves 6 days after agroinfiltration, whereas coexpression of VdCUT11 and green fluorescent protein (GFP) resulted in a normal cell death response (Fig. 6A). Conductivity assays of the coinfiltration areas of $N$. benthamiana leaves revealed that electrolyte leakage occurred 3 days after coagroinfiltration of VdCUT11 and GFP, but that the electrolyte leakage was significantly less after coagroinfiltration of VdCUT11 and VdCBM1 (Fig. 6B). Immunoblotting analysis performed on total protein extracts of the coinfiltration areas confirmed the presence of VdCUT11 in plant tissue after coexpression with VdCBM1 (Fig. 6C). Furthermore, VdCBM1 effectively suppressed the VdCUT11-triggered ROS accumulation in N. benthamiana (Fig. 6D). These results suggested that the defense response triggered by VdCUT11 can be suppressed by the $V$. dahliae immunity suppressor VdCBM1.

\section{VdCUT11 contributes to the virulence of Verticillium dahliae Vd991 on cotton cv. Junmian 1.}

To explore the possible contribution of VdCUT11 to virulence in $V$. dahliae, we constructed a VdCUT11 gene deletion mutant by replacing the coding sequence with a hygromycin resistance cassette through homologous recombination (Supplementary Fig. S2) and generated the complementary transformants by reintroducing VdCUT11 with the native promoter. All strains were verified by PCR and two were selected for infection experiments of cotton plants, using a root-dip method. The pathogenicity tests showed that VdCUT11 deletion strains were significantly less virulent toward the susceptible cotton plants 21 days after inoculation, which typically display Verticillium wilt symptoms of necrosis, wilting, and vascular discoloration when inoculated with the wild-type strain Vd991 (Fig. 7A). Transformants recovered the high virulence phenotype on cotton after reintroduction of VdCUT11 into the gene deletion strain (Fig. 7A). Fungal biomass assays confirmed that the target gene deletion mutant biomass was significantly less than the biomass of the wild type in inoculated cotton plants. The biomass of complementary transformants and the wild-type strain were comparable (Fig. 7B). Determination of the growth phenotype showed that targeted deletion of VdCUT11 does not affects radial growth and colony morphology (Supplementary Fig. S4), which suggested that VdCUT11 contributes to virulence but is not required for normal morphological development. In addition, the expression of VdCUT11 in Vd991 was significantly up-regulated (more than a threefold change) during cotton infection at one to two days after inoculation (Fig. 7C). These results suggested that VdCUT11 is a major virulence factor involved in $V$. dahliae pathogenicity, although it can be recognized and trigger plant defense responses in cotton in the absence of VdCBM1.

\section{DISCUSSION}

Cutinases play important roles in fungal-plant pathogen interactions, such as degrading plant cuticle or suberin polymers, eliciting host signals, fungal spore attachment to plant surfaces, and carbon acquisition during saprophytic growth (Cheung et al. 2009; Deising et al. 1992; Järvinen et al. 2009; Kolattukudy 1985; Kolattukudy et al. 1995; Köller et al. 1991; Skamnioti and Gurr 2007). In this study, we present the genetic evidence that the $V$. dahliae cutinase family member VdCUT11 can induce immune responses mediated by the leucine-rich repeat (LRR)-RLP/SOBIR1/BAK1 complex in $N$. benthamiana and that defense responses are dependent on the enzymatic activity or VdCUT11. Furthermore, the current study also presents evidence that the CBM1 protein VdCBM1 acts to suppress the immunity triggered by VdCUT11, facilitating the establishment of fungal infections in $N$. benthamiana. In addition, VdCUT11 contributes to virulence of $V$. dahliae on cotton, a host of major economic importance.

Many fungi produce plant cell wall-degrading enzymes for penetration and successful infection of their hosts, which are considered critical for the generation of disease symptoms and pathogenesis (Glass et al. 2013; King et al. 2011; Kubicek et al. 2014). To overcome the first barrier to plant entry, pathogens generally produce cutinases that catalyze the degradation of the cuticle and facilitate penetration (Kontkanen et al. 2009; Li et al. 2003; Lin and Kolattukudy 1980; Trail and Köller 1994; Skamnioti and Gurr 2007; van der Vlugt-Bergmans et al. 1997). Previous studies have demonstrated that several pathogens encode multiple cutinase genes to degrade different types of plant polymers (Chen et al. 2013; Skamnioti et al. 2008; Zhao et al. 2014). It has been postulated that the expansion of the cutinase family is probably associated with pathogens that possess a broad host range (Zhao et al. 2014).

In this study, we found that the $V$. dahliae $\mathrm{Vd} 991$ genome encodes 13 cutinase family members, which is a relatively high number, based on a comparative study (Zhao et al. 2014), and may help explain the broad host range of $V$. dahliae that includes hundreds of dicotyledonous hosts in at least 14 plant families (Barbara and Clewes 2003; Inderbitzin et al. 2011). Although the $V$. dahliae cutinases shared highly conserved motifs with cutinases from other phytopathogens (Chen et al. 2013), the $V$. dahliae sequences showed significant divergence and two of them (VdCUT2 and VdCUT3) even lacked a conserved motif due to the truncation in the C-terminus (Fig. 1A). In addition, phylogenetic analysis showed that the cutinases of $V$. dahliae can be divided into three groups, similarly to the cutinase family in Curvularia lunata (Liu et al. 2016), which may be associated with the functional diversity of cutinases in $V$. dahliae. We hypothesized that the cutinase family members play various roles in pathogenesis and contribute to the broad host range of $V$. dahliae, in which the expression of several VdCUTs were significantly induced during infection on cotton (Supplementary Fig. S5), though only VdCUT11 was explored in the current study. The biological functions of other V. dahliae cutinases such as VdCUT6, VdCUT7, VdCUT8, and VdCUT13, which were most similar to VdCUT11, remain to be elucidated.

Several tangible lines of evidence support functional divergence of gene family members in fungal-host interactions (Chen et al. 2013). For example, cutinases are required for spore adhesion and appressorium differentiation (Deising et al. 1992; Pascholati et al. 1992, 1993; Skamnioti and Gurr 2007), are involved in host penetration and are required for virulence (Skamnioti and Gurr 2007), and play a role in carbon acquisition during subcuticular growth of Venturia inaequalis (Köller et al. 1991). In addition to these functions, cutinases may also trigger defense responses in plants. Purified cutinase from Venturia inaequalis triggered defense responses that prevented the development of web blight symptoms on bean leaves after inoculation with Rhizoctonia spp. (Parker and Köller 1998). 
Similarly, the Sclerotinia sclerotiorum cutinase SsCUT induced defense responses in plants (Zhang et al. 2014). Likewise, in the current study VdCUT11 triggered several defense responses in $N$. benthamiana (Figs. 2 and 4).

A previous study showed that the cutinase SsCUT triggers plant defense responses independent of its enzymatic activity, as the noncatalytic $\mathrm{C}$-terminal half of the protein was sufficient to induce cell death in $N$. benthamiana. Site-directed mutagenesis of Try or Ser residues in a conserved motif of SsCUT resulted in the loss of cell death-inducing activity, providing evidence that the Try and Ser residues are the key epitopes within the conserved motif (GYSQG) (Zhang et al. 2014). In contrast to SsCUT, site-directed mutagenesis of VdCUT11 indicated that the enzymatic activity of VdCUT11 was required for triggering defense responses in $N$. benthamiana (Fig. 3), whereas the Ser residue in the conserved motif of VdCUT11 was not (Supplementary Fig. S3). The localization of VdCUT11 to the apoplast was necessary for triggering defense responses, as were the three important enzymatic activity sites (Fig. 3). Therefore, we suggest that the immunity triggered by VdCUT11 is likely mediated by the degradation of plant cell-wall polymers that results in the release of plant cell-wall fragments (DAMPs), which in turn, trigger defense responses in the plant.

The plant cell wall may be breached by pathogen-secreted lytic enzymes or mechanical damage, resulting in the generation of molecules that function as DAMPs. For example, cutin monomers released from the plant cell wall during infection of fungal foliar pathogens can act as DAMPs (Kauss et al. 1999; Schweizer et al. 1996b). DAMPs that appear in the apoplast and activate immune responses (De Lorenzo et al. 2011; Wu and Zhou 2013; Zipfel 2014) include systemin (Schilmiller and Howe 2005), OGs (Brutus et al. 2010), Peps (Huffaker et al. 2006; Yamaguchi et al.
2010). For instance, the endo-polygalacturonases may release fragments of the OGs by hydrolyzing homogalacturonan, that elicit defense responses in many plants (De Lorenzo et al. 2001).

Only a handful of PRR-ligand pairs that act in the recognition of DAMPs have been established. These include WAK1 (Cabrera et al. 2008; Decreux and Messiaen 2005; Decreux et al. 2006), PEPR (Huffaker et al. 2006; Yamaguchi et al. 2010), and a 160-kDa systemin cell-surface receptor (SR160) (Constabel et al. 1998). WAK1 is known to recognize OGs based on observations of RLKs binding OGs and pectin in vitro (Cabrera et al. 2008; Decreux and Messiaen 2005; Decreux et al. 2006). In addition, several coreceptors have been shown to be necessary for the function of receptor complexes that mediate DAMP-triggered immunity, including BAK1, BKK1, and BIK1 (Gravino et al. 2017; Liu et al. 2013; Schulze et al. 2010; Yamada et al. 2016). In this study, tobacco SERK3/BAK1 was required for VdCUT11-induced cell death and DAMPtriggered immunity (Fig. 5), similar to the DAMPs/AtPeps system, in which LRR-RLK receptors PEPR1 and PEPR2 interact with BAK1 (Gravino et al. 2017; Wu and Zhou 2013). Interestingly, VdCUT11 lost the ability to trigger cell death in NbSOBIRl-silenced plants (Fig. 5). SOBIR1 appears to be specifically required for the function of receptor complexes containing RLPs, due to the lack of an intracellular signaling domain (Liebrand et al. 2013), suggesting that DAMPs caused by the activity of VdCUT11 triggered the defense responses mediated by the LRR-RLP/SOBIR1/BAK1 complex.

$V$. dahliae is a soil-borne vascular wilt fungal plant pathogen that infects host roots and proliferates in vascular tissues (Klosterman et al. 2011). In general, roots do not contain cutin but, instead, contain the related compound suberin (Murray et al. 2013). In contrast to the cuticle that is strictly deposited on
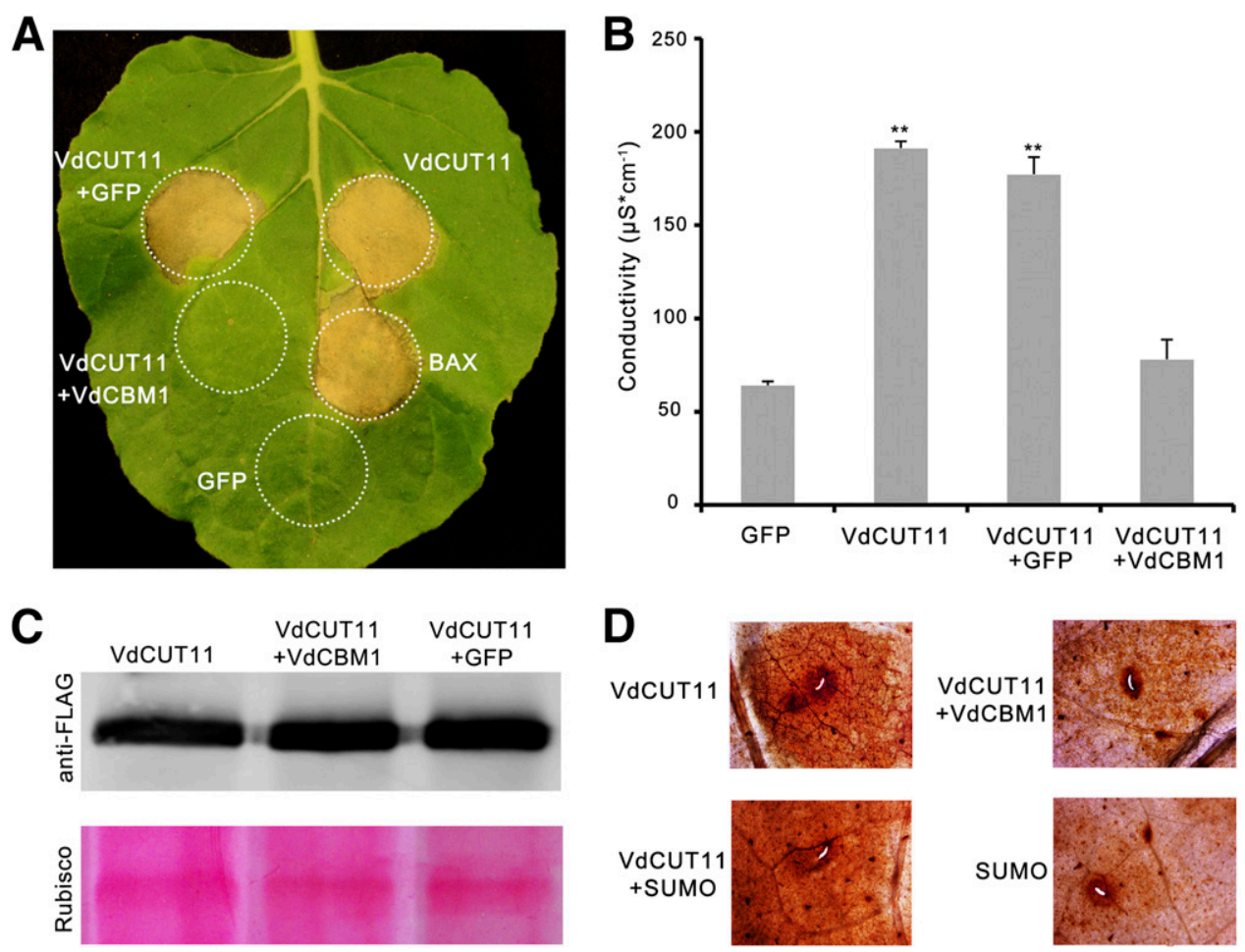

Fig. 6. The cell death-inducing activity of VdCUT11 is suppressed by the CBM1 protein VdCBM1 in Nicotiana benthamiana. A, Assay for suppression of cell death-inducing activity of VdCBM1 by coinfiltration with VdCUT11 in 4-week-old $N$. benthamiana leaves 6 days after agroinfiltration. BAX (Bcl-2associated X) and green fluorescent protein (GFP) were used as a positive and negative control, respectively. B, Electrolyte leakage assay in 4-week-old $N$. benthamiana leaves $48 \mathrm{~h}$ after coinfiltration of VdCUT11 with VdCBM1. C, Immunoblotting analysis of proteins in N. benthamiana leaves transiently expressing VdCUT11 after coinfiltration with VdCBM1. Ponceau S-stained Rubisco protein is shown as a total protein loading control. D, Reactive oxygen species accumulation in 4-week-old $N$. benthamiana leaves $12 \mathrm{~h}$ after coinfiltration of VdCUT11 with VdCBM1 recombinant proteins. Leaves were stained with 3'3-diaminobenzidine. 
the outer surface of epidermal cells, suberin is deposited as a layer or a multilayered lamella on the inner surface of the cell wall of suberizing cells or within primary cell walls (Casparian strips or epidermal cells) (Enstone et al. 2002; Karahara et al. 2004; Ranathunge et al. 2008; Thomas et al. 2007). Unlike the extremely early (3-h postinoculation) upregulation of $\mathrm{ClCUT7}$ involved in cuticle degradation (Liu et al. 2016), the transcript level of VdCUT11 increased slowly and was most significantly up-regulated during the infection stage (one to two days) (Fig. 7C). Thus, we suggest that VdCUT11 is involved in the degradation of suberin in roots, which results in the release of plant cell-wall fragments (DAMPs).

Not all fungal cutinases have been conclusively implicated in pathogenicity and virulence (Dantzig et al. 1986; Liu et al. 2016; Skamnioti and Gurr 2007; Sweigard et al. 1992). For example, the virulence of a $M$. oryzae cutinase2-deficient mutant was reduced but could be restored by complementation with the native cutinase gene (Skamnioti and Gurr 2007). Moreover, the deletion of $M$. oryzae CUT1 did not alter the virulence of this fungus on rice (Sweigard et al. 1992). In the current study, the deletion of VdCUT11 significantly reduced the virulence of Vd991 in cotton cv. Junmian 1 (Fig. 7A and B). VdCUT11 appears to be a major virulence factor due to its enzymatic function, although it also can trigger plant defenses, depending on the host genotype, similar to the GH12 proteins in fungi and oomycetes (Gui et al. 2017; Ma et al. 2015). In addition, despite the ability of plants to recognize and respond to pathogen effectors, pathogens have clearly evolved mechanisms to surmount the plant defense responses (Jones and Dangl 2006). A previous study showed that the $V$. dahliae effector VdCBM1 suppresses the GH12 protein-triggered immunity mediated by PRRs in N. benthamiana (Gui et al. 2017). Interestingly, we found that VdCBM1 also suppresses the defense DAMP-triggered responses induced by VdCUT11 (Fig. 6) and, thus, facilitates host infection and colonization.

\section{MATERIALS AND METHODS}

\section{Identification of cutinase gene family members in V. dahliae Vd991.}

Candidate cutinase gene family members were predicted by HMMER, using the HMM profile of the CE5 domain (PF01083) against the $V$. dahliae Vd991 genome (the Whole Genome Shotgun project has been deposited at DDBJ/ENA/GenBank under accession NVYA00000000 [Chen et al. 2017]) using the default parameters. Returned hits with $E$ values $<1 \mathrm{e}-10$ and scores $>30$ were then manually selected. Secreted proteins were identified using four programs commonly used to identify protein localization, as described previously (Klosterman et al. 2011). Putative extracellular proteins containing a signal peptide

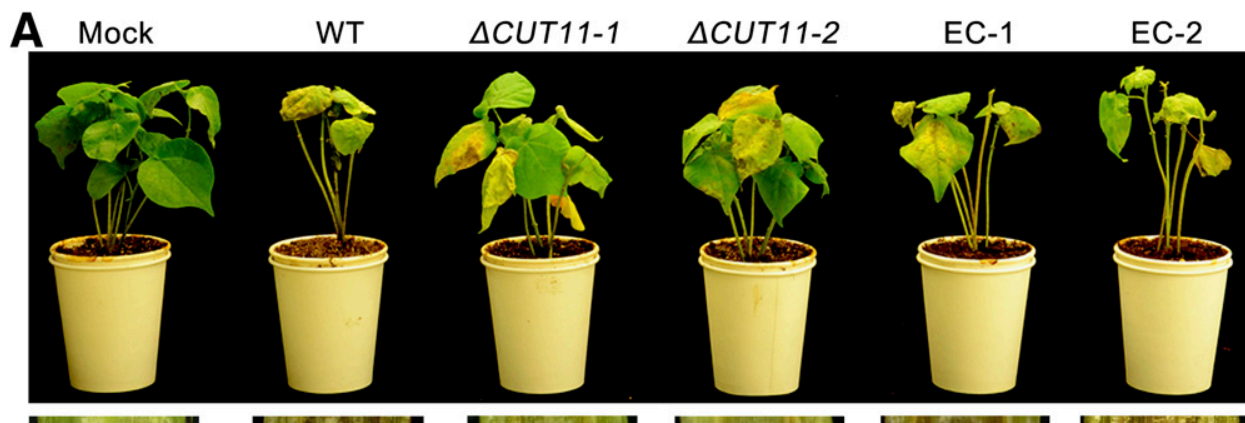

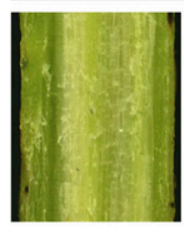

B

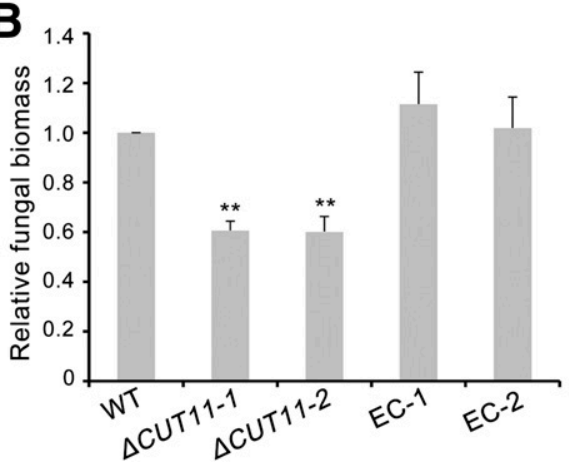

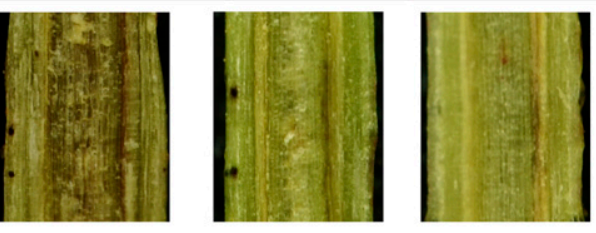

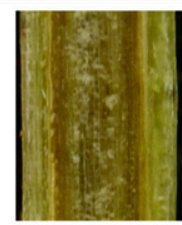

C

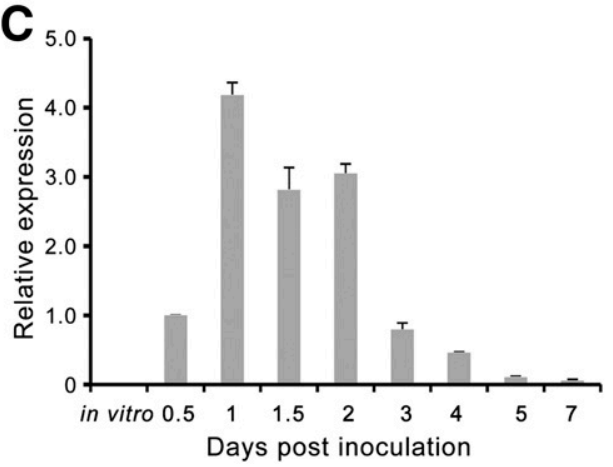

Fig. 7. Detection of the virulence function of VdCUT11 during cotton pathogenesis. A, Phenotypes of cotton seedlings inoculated with Verticillium dahliae Vd991 VdCUT11 gene-deletion strains and complementary transformants. Two-week-old seedlings of susceptible cotton (cv. Junmian 1) were inoculated with sterile water (Mock), wild-type $V$. dahliae (WT), VdCUT11 gene deletion strains ( $\Delta$ CUT11-1 and $\Delta$ CUT11-2), and complementary transformants (EC-1 and EC-2). Verticillium wilt symptoms (top) were photographed three weeks after inoculation, and discoloration in shoot longitudinal sections is shown (bottom). B, In planta fungal biomass quantification of gene-deletion strains and corresponding ectopic transformants inoculated on cotton were determined by quantitative polymerase chain reaction (qPCR). Error bars represent standard errors. Asterisks $(* *)$ indicate significant differences $(P<0.01)$, according to unpaired Student's $t$ tests. C, Expression of VdCUT11 during infection of cotton roots. Two-week-old cotton plants (cv. Junmian 1) were inoculated with wildtype $V$. dahliae Vd991 and were harvested at different timepoints after inoculation. Quantitative reverse transcription PCR was performed to determine the expression levels of VdCUT11 relative to $\beta$-tubulin (VDAG_10074). Expression of genes during growth of V. dahliae Vd991 on Czapek medium was used as a control. 
but lacking transmembrane domains were identified as secreted proteins. The orthologous genes in the $V$. dahliae VdLs.17 genome were determined by using BLAST (Altschul et al. 1997). Clustal X2 was used for multiple sequence alignment (Larkin et al. 2007). Conserved motifs and enzymatic activity sites were determined according to a PROSITE profile (PDOC00140). An unrooted tree was generated in the MEGA6 program, using the maximum-likelihood method with 1,000 bootstrap replicates (Tamura et al. 2013).

\section{Transient gene-expression assay.}

Transient gene-expression assays were performed in $N$. benthamiana, using a total of 20 wild-type and mutant genes including i) the full-length coding sequences of cutinase family genes VdCUT1 to VdCUT13 (Supplementary Table S4), ii) VdCUT11 ${ }^{\text {-SP }}$, a mutant of VdCUT11 missing the sequence encoding the signal peptide, iii) $\mathrm{EG} 3^{\mathrm{SP}}-\mathrm{VdCUT} 11^{\text {-SP }}$, a chimeric sequence of VdCUT1 $1^{\text {-SP }}$ and the VdEG3 signal peptide, iv) four site-directed catalytic residue mutants of VdCUT11 (VdCUT11 ${ }^{S M}$, S133A, D198A, and H210A), and v) the immune suppressor VdCBM1. All sequences contained a $3^{\prime}$ FLAG tag and were separately cloned into the PVX vector pGR107 and transformed into Agrobacterium tumefaciens GV3101. Agroinfiltration assays were performed on $N$. benthamiana plants using the BAX and GFP as positive and negative controls, respectively. Each assay was performed on six leaves from three individual plants and was repeated at least three times. The suppression of cell-death induction by VdCBM1 was tested by coinfiltration with VdCUT11. Phenotypes were photographed 6 days after infiltration. Total proteins were extracted using a P-PER plant protein extraction kit and protease inhibitor cocktail kit (Thermo Scientific, Waltham, MA, U.S.A.) from agroinfiltrated $N$. benthamiana leaves $60 \mathrm{~h}$ after infiltration. Transient protein expression in $N$. benthamiana was assessed using anti-FLAG antibodies (Sigma-Aldrich, St. Louis).

\section{Recombinant protein purification and enzyme activity assays.}

The wild-type gene VdCUT11 and site-directed mutagenized gene $V d C U T 11^{S M}$ were cloned and were ligated into pSMARTI vector with a SUMO tag fused in-frame to the $\mathrm{N}$ terminus. After transformation into Escherichia coli BL21(DE3)pLysS, recombinant protein expression was induced by adding $1 \mathrm{mM}$ isopropyl- $\beta$-D-thiogalactoside for $8 \mathrm{~h}$ at $15^{\circ} \mathrm{C}$. The cells were lysed by freezing and thawing followed by sonication, and the recombinant protein was purified as previously described for other His $_{6}$-SUMO-tagged proteins (Rao et al. 2014). The cleared lysate was incubated with nickel-NTA resin for 1 to $2 \mathrm{~h}$ at $4^{\circ} \mathrm{C}$ with mixing and the tagged proteins eluted batchwise with lysis buffer plus $300 \mathrm{mM}$ imidazole. Enzyme activities were measured as described previously (Chen et al. 2008) in 1-ml final volumes containing pNPB $(1 \mathrm{mM})$, the enzyme, and the assay buffer $(20 \mathrm{mM}$ Tris- $\mathrm{HCl}, 10 \mathrm{mM} \mathrm{NaCl}$, and $50 \mathrm{mM}$ sodium taurodeoxycholate, $\mathrm{pH} 8.0$ ) at $37^{\circ} \mathrm{C}$, with the substrates of pNPB. The hydrolysis of pNPB was spectrophotometrically monitored for the formation of $p$-nitrophenol at $405 \mathrm{nM}$. One unit of enzyme activity was defined as the production of $1 \mu \mathrm{M}$ of $p$-nitrophenol per minute. Unpaired Student's $t$ tests were performed to determine statistical significance, and $P$ values $<0.01$ between the two treatments groups were considered statistically significant.

\section{Yeast signal sequence trap system.}

Functional validation of the predicted signal peptide was performed as described previously (Jacobs et al. 1997). The predicted signal peptide sequence of $V d C U T 11$ was fused in-frame to the secretion-defective invertase gene in the vector pSUC2 and was transformed into the yeast strain YTK12. YTK12 transformed with the functional signal peptide of Avr1b or an empty pSUC2 vector were used as positive and negative controls, respectively. Positive clones were confirmed by PCR using vector-specific primers. All the strains were screened on CMD-W (lacking tryptophan) medium and the positive transformants were incubated on YPRAA medium (2\% raffinose) (Liu et al. 2013).

\section{ROS activity, electrolyte leakage, and the suppression of phytopathogen infection.}

Recombinant proteins $(5 \mu \mathrm{M})$ were used for detecting cell death-inducing activity in 4-week-old plants of $N$. benthamiana, 2 -week-old plants of cotton cv. Junmian 1 cotyledons, and 2-week-old plants of cv. MoneyMaker tomato. The cell-death response in roots was detected by the 2-day-old cotton hypotocotyls absorbing $20 \mu \mathrm{l}$ of $5 \mu \mathrm{M}$ recombinant proteins, and the phenotypes were investigated $48 \mathrm{~h}$ after treatment with the recombinant proteins. ROS generation was detected using DAB solution (Solarbio, Beijing). Briefly, $400 \mathrm{nM}$ recombinant proteins were infiltrated into the 4-week-old $N$. benthamiana seedling leaves and were collected $12 \mathrm{~h}$ after infiltration. The leaves were collected, were placed in DAB, were vacuum infiltrated until the leaves were saturated, and were incubated for $8 \mathrm{~h}$ in the dark at $25^{\circ} \mathrm{C}$. Subsequently, the leaves were cleared with $75 \%$ ( $\mathrm{vol} / \mathrm{vol})$ ethanol until the chlorophyll content was completely removed, and stereomicroscopic images were captured (M205C; Leica, Bensheim, Germany).

Electrolyte leakage assays for $N$. benthamiana were modified, as follows, from previously described procedures (Oh et al. 2010). Briefly, nine leaf discs (1 cm in diameter) were harvested from the Agrobacterium-infiltrated regions of nine leaves of three plants and incubated in $9 \mathrm{ml}$ of deionized water with shaking at $165 \mathrm{rpm}$ at $25^{\circ} \mathrm{C}$ for $2 \mathrm{~h}$. Ion conductivity was then measured using a conductivity meter with Probe LE703 (Mettler-Toledo, Shanghai, China). The assays were repeated three times.

Recombinant protein VdCUT11 and site-directed mutagenized VdCUT11 ${ }^{\mathrm{SM}}(400 \mathrm{nM})$ were separately syringe-infiltrated into the whole area of leaves of 5-week-old $N$. benthamiana plants. At $12 \mathrm{~h}$ after infiltration, $5 \mu \mathrm{l}$ of $2 \times 10^{6}$-conidia/ml solutions of B. cinerea or P. capsici was placed on the infiltrated area. The inoculated plants were placed in a light incubator under a 16 -h photoperiod $\left(60 \mu \mathrm{mol}^{-2} \mathrm{~s}^{-1}\right)$ at $25^{\circ} \mathrm{C}$ and $80 \%$ relative humidity. Three leaves per plant were inoculated per experiment and each experiment was repeated three times.

\section{VIGS in $N$. benthamiana.}

VIGS assays based on recombinant TRV were performed as described previously (Liu et al. 2002). The plasmid constructs pTRV1, pTRV2::BAK1, and pTRV2::SOBIR1 were introduced into A. tumefaciens. Agrobacterium strains harboring either the pTRV2::BAK1 or pTRV2::SOBIR1 plasmid were combined with strains harboring the pTRV1 vector in a 1:1 ratio and were coinfiltrated into two primary leaves of 4-week-old $N$. benthamiana plants. Plasmid pTRV2:GFP was used as the control. The effectiveness of the VIGS assay was evaluated using the PDS (phytoene desaturase) gene as previously described (Liu et al. 2002). The silencing efficiencies of $N b B A K 1$ and $\mathrm{NbSOBIRl}$ were validated by qRT-PCR analysis of RNA. The experiment was performed three times with six plants for each TRV construct per experiment.

\section{Fungal transformations.}

Targeted gene-deletion constructs were generated based on a previously described method (Liu et al. 2013). Briefly, approximately $1-\mathrm{kb}$ flanking sequences of targeted genes were amplified from V. dahliae Vd991 genomic DNA and were fused 
to a $1.8-\mathrm{kb}$ hygromycin-resistant cassette. The fusion fragment was introduced into the binary vector pGKO2-Gateway, using a standard BP reaction. To reintroduce the gene into the target gene-deletion strain, the wild-type gene VdCUT11 was cloned into the donor vector pCT-HN (Liu et al. 2013). The targeted gene-deletion strains and complementation transformants were obtained using a previously described Agrobacteriummediated transformation method (Liu et al. 2013), using $V$. dahliae $\mathrm{Vd} 991$. The positive targeted gene deletion of $V d C U T 11$ was identified using PCR with two primer pairs, a $H y g$-specific primer pair and the flanking test primer pair of VdCUT11.

\section{Pathogenicity assays.}

To test the pathogenicity function of VdCUT11, all the strains, including VdCUT11 targeted deletion strains, complementary transformants, and the wild-type strain Vd991 were cultured on potato dextrose agar medium or in liquid Czapek medium for 7 days at $25^{\circ} \mathrm{C}$. Cotton (Gossypium hirsutum cv. Junmian 1) plants were grown at 23 and $27^{\circ} \mathrm{C}$, respectively, in a greenhouse (14-h day and 10-h night cycle). Two-week-old cotton seedlings were inoculated with $5 \times 10^{6}$ conidia per milliliter by the root-dip method (Wu and Zhou 2013). Disease symptoms on inoculated plants were observed at 21 days postinoculation on cotton. Vascular discoloration of cotton was observed by cutting the cotton shoot at 3 weeks after inoculation. Fungal biomass in infected cotton plants was determined as previously described (Santhanam et al. 2013). qPCR was performed using the qPCR SYBR premix Ex Taq II kit (TaKaRa, Tokyo) with primers of the cotton $18 S$ gene. Unpaired Student's $t$ tests were performed to determine statistical significance at $P$ values $<0.05$ between two treatment groups.

\section{RNA extraction and qRT-PCR.}

The treatment samples of $N$. benthamiana were collected $24 \mathrm{~h}$ after infiltration with $400 \mathrm{nM}$ recombinant VdCUT11 protein. Cotton plants were inoculated using a root-dip method in a $5 \times 10^{6}$-conidia/ml solution and collections were gathered at different timepoints. The AxyPreP multisource total RNA miniprep kit (Axygen, Union City, CA, U.S.A.) was used to isolate total RNA, and first-stand cDNA was synthesized using a RevertAid first cDNA synthesis kit according to the manufacturer's instructions (Thermo, Vilnius, Lithuania). qRT-PCR was performed under the following conditions: an initial $95^{\circ} \mathrm{C}$ denaturation step for $10 \mathrm{~min}$, followed by 40 cycles of $95^{\circ} \mathrm{C}$ for $15 \mathrm{~s}$ and $60^{\circ} \mathrm{C}$ for $1 \mathrm{~min}$. The tobacco or $V$. dahliae $E F-1 \alpha$ were used as endogenous references. Relative transcript levels among various samples were determined using the $2^{-\Delta \Delta \mathrm{CT}}$ method with three independent determinations (Livak et al. 2001). Unpaired Student's $t$ tests were performed to determine statistical significance, and $P$ values $<0.05$ between two treatments groups were considered statistically significant.

\section{ACKNOWLEDGMENTS}

This work was supported by the China Natural Scientific Foundation (numbers 31671986, 31200113, and 31171799), the National Key Research and Development Program of China (2017YFD0201900, 2017YFD0200601), the CAAS (an Agricultural Science and Technology Innovation Program grant to X.-F. Dai), the Fundamental Research Funds for Central Non-Profit Scientific Institution (Y2016CG11, S2016JC05, S2016CG01), the Special Public Welfare Industry Research on Agriculture (201503109).

\section{LITERATURE CITED}

Altschul, S. F., Madden, T. L., Schäffer, A. A., Zhang, J., Zhang, Z., Miller, W., and Lipman, D. J. 1997. Gapped BLAST and PSI-BLAST: A new generation of protein database search programs. Nucleic Acids Res. 25: 3389-3402.
Barbara, D. J., and Clewes, E. 2003. Plant pathogenic Verticillium species: How many of them are there? Mol. Plant Pathol. 4:297-305.

Bessire, M., Chassot, C., Jacquat, A. C., Humphry, M., Borel, S., Petétot, J. M., Métraux, J. P., and Nawrath, C. 2007. A permeable cuticle in Arabidopsis leads to a strong resistance to Botrytis cinerea. EMBO J. 26:2158-2168.

Boller, T., and Felix, G. 2009. A renaissance of elicitors: Perception of microbe-associated molecular patterns and danger signals by patternrecognition receptors. Annu. Rev. Plant Biol. 60:379-406.

Brutus, A., Sicilia, F., Macone, A., Cervone, F., and De Lorenzo, G. 2010. A domain swap approach reveals a role of the plant wall-associated kinase 1 (WAK1) as a receptor of oligogalacturonides. Proc. Natl. Acad. Sci. U.S.A. 107:9452-9457.

Cabrera, J. C., Boland, A., Messiaen, J., Cambier, P., and Van Cutsem, P. 2008. Egg box conformation of oligogalacturonides: The timedependent stabilization of the elicitor-active conformation increases its biological activity. Glycobiology 18:473-482.

Carvalho, C. M., Aires-Barros, M. R., and Cabral, J. M. 1999. Cutinase: From molecular level to bioprocess development. Biotechnol. Bioeng. 66:17-34.

Chasan, R. 1992. Cutinase: Not a weapon in fungal combat? Plant Cell 4: 617-618.

Chassot, C., and Metraux, J. P. 2005. The cuticle as source of signals for plant defense. Plant Biosyst. 139:28-31.

Chassot, C., Nawrath, C., and Métraux, J. P. 2007. Cuticular defects lead to full immunity to a major plant pathogen. Plant J. 49:972-980.

Chen, J. Y., Liu, C., Gui, Y. J., Si, K. W., Zhang, D. D., Wang, J, Short, D. P. G, Huang, J. Q., Li, N. Y., Liang, Y., Zhang, W. Q., Yang, L., Ma X. F., Li, T. G., Zhou, L., Wang, B. L., Bao, Y. M., Subbarao, K. V. Zhang, G. Y., and Dai, X. F. 2017. Comparative genomics reveals cottonspecific virulence factors in flexible genomic regions in Verticillium dahliae and evidence of horizontal gene transfer from Fusarium. New Phytol. Published online. doi.org/10.1111/nph.14861.

Chen, J. Y., Xiao, H. L., Gui, Y. J., Zhang, D. D., Li, L., Bao, Y. M., and Dai, X. F. 2016. Characterization of the Verticillium dahliae exoproteome involves in pathogenicity from cotton-containing medium. Front. Microbiol. 7:1709.

Chen, S., Su, L., Chen, J., and Wu, J. 2013. Cutinase: Characteristics, preparation, and application. Biotechnol. Adv. 31:1754-1767.

Chen, S., Tong, X., Woodard, R. W., Du, G., Wu, J., and Chen, J. 2008. Identification and characterization of bacterial cutinase. J. Biol. Chem. 283:25854-25862.

Cheung, I. W., Nakayama, S., Hsu, M. N., Samaranayaka, A. G., and LiChan, E. C. 2009. Angiotensin-I converting enzyme inhibitory activity of hydrolysates from oat (Avena sativa) proteins by in silico and in vitro analyses. J. Agric. Food Chem. 57:9234-9242.

Choi, H. W., and Klessig, D. F. 2016. DAMPs, MAMPs, and NAMPs in plant innate immunity. BMC Plant Biol. 16:232.

Constabel, C. P., Yip, L., and Ryan, C. A. 1998. Prosystemin from potato, black nightshade, and bell pepper: Primary structure and biological activity of predicted systemin polypeptides. Plant Mol. Biol. 36:55-62.

Dantzig, A. H., Zuckerman, S. H., and Andonov-Roland, M. M. 1986. Isolation of a Fusarium solani mutant reduced in cutinase activity and virulence. J. Bacteriol. 168:911-916.

Davies, K. A., DeLorono, I., Foster, S. J., Li, D., Johnstone, K., and Ashby, A. M. 2000. Evidence for a role of cutinase in pathogenicity of Pyrenopeziza brassicae on brassicas. Physiol. Mol. Plant Pathol. 57: 63-75.

De Lorenzo, G., Brutus, A., Savatin, D. V., Sicilia, F., and Cervone, F. 2011. Engineering plant resistance by constructing chimeric receptors that recognize damage-associated molecular patterns (DAMPs). FEBS Lett 585:1521-1528.

De Lorenzo, G., D'Ovidio, R., and Cervone, F. 2001. The role of polygalacturonase-inhibiting proteins (PGIPs) in defense against pathogenic fungi. Annu. Rev. Phytopathol. 39:313-335.

Decreux, A., and Messiaen, J. 2005. Wall-associated kinase WAK1 interacts with cell wall pectins in a calcium-induced conformation. Plant Cell Physiol. 46:268-278.

Decreux, A., Thomas, A., Spies, B., Brasseur, R., Van Cutsem, P., and Messiaen, J. 2006. In vitro characterization of the homogalacturonanbinding domain of the wall-associated kinase WAK1 using site-directed mutagenesis. Phytochemistry 67:1068-1079.

Deising, H., Nicholson, R. L., Haug, M., Howard, R. J., and Mendgen, K. 1992. Adhesion pad formation and the involvement of cutinase and esterases in the attachment of uredospores to the host cuticle. Plant Cell 4:1101-1111.

Dickman, M. B., and Patil, S. S. 1986. A rapid and sensitive plate assay for the detection of cutinase produced by plant pathogenic fungi. Phytopathology 76:473-475. 
Egmond, M. R., and de Vlieg, J. 2000. Fusarium solani pisi cutinase. Biochimie 82:1015-1021

Enstone, D. E., Peterson, C. A., and Ma, F. 2002. Root endodermis and exodermis: Structure, function, and responses to the environment. J. Plant Growth Regul. 21:335-351.

Gaulin, E., Dramé, N., Lafitte, C., Torto-Alalibo, T., Martinez, Y., AmelineTorregrosa, C., Khatib, M., Mazarguil, H., Villalba-Mateos, F., Kamoun, S., Mazars, C., Dumas, B., Bottin, A., Esquerré-Tugayé, M. T., and Rickauer, M. 2006. Cellulose binding domains of a Phytophthora cell wall protein are novel pathogen-associated molecular patterns. Plant Cell 18:1766-1777.

Glass, N. L., Schmoll, M., Cate, J. H., and Coradetti, S. 2013. Plant cell wall deconstruction by ascomycete fungi. Annu. Rev. Microbiol. 67:477-498

Gravino, M., Locci, F., Tundo, S., Cervone, F., Savatin, D. V., and De Lorenzo, G. 2017. Immune responses induced by oligogalacturonides are differentially affected by AvrPto and loss of BAK1/BKK1 and PEPR1/PEPR2. Mol. Plant Pathol. 18:582-595.

Gui, Y. J., Chen, J. Y., Zhang, D. D., Li, N. Y., Li, T. G., Zhang, W. Q., Wang, X. Y., Short, D. P. G., Li, L., Guo, W., Kong, Z. Q., Bao, Y. M., Subbarao, K. V., and Dai, X. F. 2017. Verticillium dahliae manipulates plant immunity by glycoside hydrolase 12 proteins in conjunction with carbohydrate-binding module 1. Environ. Microbiol. 19:1914-1932.

Huffaker, A., Pearce, G., and Ryan, C. A. 2006. An endogenous peptide signal in Arabidopsis activates components of the innate immune response. Proc. Natl. Acad. Sci. U.S.A. 103:10098-10103.

Inderbitzin, P., Bostock, R. M., Davis, R. M., Usami, T., Platt, H. W., and Subbarao, K. V. 2011. Phylogenetics and taxonomy of the fungal vascular wilt pathogen Verticillium, with the descriptions of five new species. PLoS One 6:e28341.

Jacobs, K. A., Collins-Racie, L. A., Colbert, M., Duckett, M., Golden-Fleet, M., Kelleher, K., Kriz, R., LaVallie, E. R., Merberg, D., Spaulding, V., Stover, J., Williamson, M. J., and McCoy, J. M. 1997. A genetic selection for isolating cDNAs encoding secreted proteins. Gene 198:289-296.

Järvinen, R., Silvestre, A. J., Holopainen, U., Kaimainen, M., Nyyssölä, A., Gil, A. M., Pascoal Neto, C., Lehtinen, P., Buchert, J., and Kallio, H. 2009. Suberin of potato (Solanum tuberosum var. Nikola): Comparison of the effect of cutinase CcCut 1 with chemical depolymerization. J. Agric. Food Chem. 57:9016-9027.

Jones, J. D., and Dangl, J. L. 2006. The plant immune system. Nature 444: 323-329.

Karahara, I., Ikeda, A., Kondo, T., and Uetake, Y. 2004. Development of the Casparian strip in primary roots of maize under salt stress. Planta 219: 41-47.

Kauss, H., Fauth, M., Merten, A., and Jeblick, W. 1999. Cucumber hypocotyls respond to cutin monomers via both an inducible and a constitutive $\mathrm{H}(2) \mathrm{O}(2)$-generating system. Plant Physiol. 120:1175-1182.

Kim, T. H., Park, J. H., Kim, M. C., and Cho, S. H. 2008. Cutin monomer induces expression of the rice OsLTP5 lipid transfer protein gene. J. Plant Physiol. 165:345-349.

King, B. C., Waxman, K. D., Nenni, N. V., Walker, L. P., Bergstrom, G. C., and Gibson, D. M. 2011. Arsenal of plant cell wall degrading enzymes reflects host preference among plant pathogenic fungi. Biotechnol. Biofuels 4:4.

Klosterman, S. J., Atallah, Z. K., Vallad, G. E., and Subbarao, K. V. 2009. Diversity, pathogenicity, and management of verticillium species. Annu. Rev. Phytopathol. 47:39-62.

Klosterman, S. J., Subbarao, K. V., Kang, S., Veronese, P., Gold, S. E., Thomma, B. P., Chen, Z., Henrissat, B., Lee, Y. H., Park, J., GarciaPedrajas, M. D., Barbara, D. J., Anchieta, A., de Jonge, R., Santhanam, P., Maruthachalam, K., Atallah, Z., Amyotte, S. G., Paz, Z., Inderbitzin, P., Hayes, R. J., Heiman, D. I., Young, S., Zeng, Q., Engels, R., Galagan, J., Cuomo, C. A., Dobinson, K. F., and Ma, L. J. 2011. Comparative genomics yields insights into niche adaptation of plant vascular wilt pathogens. PLoS Pathog. 7:e1002137.

Kolattukudy, P. E. 1985. Enzymatic penetration of the plant cuticle by fungal pathogens. Annu. Rev. Phytopathol. 23:223-250.

Kolattukudy, P. E., Li, D. X., Hwang, C. S., and Flaishman, M. A. 1995. Host signals in fungal gene-expression involved in penetration into the host. Can. J. Bot. 73(S1):S1160-S1168.

Köller, W., Parker, D. M., and Becker, C. M. 1991. Role of cutinase in the penetration of apple leaves by Venturia inaequalis. Phytopathology 81: 1375-1379.

Kontkanen, H., Westerholm-Parvinen, A., Saloheimo, M., Bailey, M. Rättö, M., Mattila, I., Mohsina, M., Kalkkinen, N., Nakari-Setälä, T., and Buchert, J. 2009. Novel Coprinopsis cinerea polyesterase that hydrolyzes cutin and suberin. Appl. Environ. Microbiol. 75:2148-2157.

Kubicek, C. P., Starr, T. L., and Glass, N. L. 2014. Plant cell wall-degrading enzymes and their secretion in plant-pathogenic fungi. Annu. Rev. Phytopathol. 52:427-451.
Larkin, M. A., Blackshields, G., Brown, N. P., Chenna, R., McGettigan, P. A., McWilliam, H., Valentin, F., Wallace, I. M., Wilm, A., Lopez, R., Thompson, J. D., Gibson, T. J., and Higgins, D. G. 2007. Clustal W and Clustal X version 2.0. Bioinformatics 23:2947-2948.

Li, D., Ashby, A. M., and Johnstone, K. 2003. Molecular evidence that the extracellular cutinase $\mathrm{Pbc} 1$ is required for pathogenicity of Pyrenopeziza brassicae on oilseed rape. Mol. Plant-Microbe Interact. 16:545-552.

Liebrand, T. W., van den Berg, G. C., Zhang, Z., Smit, P., Cordewener, J. H., America, A. H., Sklenar, J., Jones, A. M., Tameling, W. I., Robatzek, S., Thomma, B. P., and Joosten, M. H. 2013. Receptor-like kinase SOBIR1/EVR interacts with receptor-like proteins in plant immunity against fungal infection. Proc. Natl. Acad. Sci. U.S.A. 110:10010-10015.

Liebrand, T. W., van den Burg, H. A., and Joosten, M. H. 2014. Two for all: Receptor-associated kinases SOBIR1 and BAK1. Trends Plant Sci. 19: 123-132.

Lin, T. S., and Kolattukudy, P. E. 1980. Isolation and characterization of a cuticular polyester (cutin) hydrolysing enzyme from phytopathogenic fungi. Physiol. Plant Pathol. 17:1-15.

Liu, S. Y., Chen, J. Y., Wang, J. L., Li, L., Xiao, H. L., Adam, S. M., and Dai, X. F. 2013. Molecular characterization and functional analysis of a specific secreted protein from highly virulent defoliating Verticillium dahliae. Gene 529: 307-316.

Liu, T., Hou, J., Wang, Y., Jin, Y., Borth, W., Zhao, F., Liu, Z., Hu, J., and Zuo, Y. 2016. Genome-wide identification, classification and expression analysis in fungal-plant interactions of cutinase gene family and functional analysis of a putative ClCUT7 in Curvularia lunata. Mol. Genet. Genomics 291:1105-1115.

Liu, Y., Schiff, M., Marathe, R., and Dinesh-Kumar, S. P. 2002. Tobacco Rarl, EDS1 and NPR1/NIM1 like genes are required for $N$-mediated resistance to tobacco mosaic virus. Plant J 30:415-429.

Liu, Z., Wu, Y., Yang, F., Zhang, Y., Chen, S., Xie, Q., Tian, X., and Zhou, J. M. 2013. BIK1 interacts with PEPRs to mediate ethylene-induced immunity. Proc. Natl. Acad. Sci. U.S.A. 110:6205-6210.

Livak, K. J., and Schmittgen, T. D. 2001. Analysis of relative gene expression data using real-time quantitative PCR and the 2(-Delta Delta C(T)) method. Methods 25:402-408.

Ma, Z., Song, T., Zhu, L., Ye, W., Wang, Y., Shao, Y., Dong, S., Zhang, Z., Dou, D., Zheng, X., Tyler, B. M., and Wang, Y. 2015. A Phytophthora sojae glycoside hydrolase 12 protein is a major virulence factor during soybean infection and is recognized as a PAMP. Plant Cell 27: 2057-2072.

Martinez, C., De Geus, P., Lauwereys, M., Matthyssens, G., and Cambillau, C. 1992. Fusarium solani cutinase is a lipolytic enzyme with a catalytic serine accessible to solvent. Nature 356:615-618.

Medzhitov, R., and Janeway, C. A., Jr. 1997. Innate immunity: The virtues of a nonclonal system of recognition. Cell 91:295-298.

Monaghan, J., and Zipfel, C. 2012. Plant pattern recognition receptor complexes at the plasma membrane. Curr. Opin. Plant Biol. 15: 349-357

Murray, J. D., Cousins, D. R., Jackson, K. J., and Liu, C. 2013. Signaling at the root surface: The role of cutin monomers in mycorrhization. Mol Plant 6:1381-1383.

Oh, C. S., Pedley, K. F., and Martin, G. B. 2010. Tomato 14-3-3 protein 7 positively regulates immunity-associated programmed cell death by enhancing protein abundance and signaling ability of MAPKKK (alpha). Plant Cell 22: 260-272.

Parker, D. M., and Köller, W. 1998. Cutinase and other lipolytic esterases protect bean leaves from infection by Rhizoctonia solani. Mol. PlantMicrobe Interact. 11:514-522.

Pascholati, S. F., Deising, H., Leite, B., Anderson, D., and Nicholson, R. L. 1993. Cutinase and nonspecific esterase activities in the conidial mucilage of Colletotrichum graminicola. Physiol. Mol. Plant Pathol. 42:37-51.

Pascholati, S. F., Yoshioka, H., Kunoh, H., and Nicholson, R. L. 1992. Preparation of the infection court by Erysiphe graminis f. sp. hordei: Cutinase is a component of the conidial exudate. Physiol. Mol. Plant Pathol. 41:53-59.

Postel, S., Küfner, I., Beuter, C., Mazzotta, S., Schwedt, A., Borlotti, A., Halter, T., Kemmerling, B., and Nürnberger, T. 2010. The multifunctional leucine-rich repeat receptor kinase BAK1 is implicated in Arabidopsis development and immunity. Eur. J. Cell Biol. 89:169-174.

Ranathunge, K., Thomas, R. H., Fang, X., Peterson, C. A., Gijzen, M., and Bernards, M. A. 2008. Soybean root suberin and partial resistance to root rot caused by Phytophthora sojae. Phytopathology 98:1179-1189.

Rao, T., Lubin, J. W., Armstrong, G. S., Tucey, T. M., Lundblad, V., and Wuttke, D. S. 2014. Structure of Est3 reveals a bimodal surface with differential roles in telomere replication. Proc. Natl. Acad. Sci. U.S.A. 111:214-218 
Rogers, L. M., Flaishman, M. A., and Kolattukudy, P. E. 1994. Cutinase gene disruption in Fusarium solani f sp pisi decreases its virulence on pea. Plant Cell 6:935-945.

Santhanam, P., van Esse, H. P., Albert, I., Faino, L., Nürnberger, T., and Thomma, B. P. H. J. 2013. Evidence for functional diversification within a fungal NEP1-like protein family. Mol. Plant Microbe Interact. 26: 278-286.

Schilmiller, A. L., and Howe, G. A. 2005. Systemic signaling in the wound response. Curr. Opin. Plant Biol. 8:369-377.

Schulze, B., Mentzel, T., Jehle, A. K., Mueller, K., Beeler, S., Boller, T., Felix, G., and Chinchilla, D. 2010. Rapid heteromerization and phosphorylation of ligand-activated plant transmembrane receptors and their associated kinase BAK1. J. Biol. Chem. 285:9444-9451.

Schweizer, P., Felix, G., Buchala, A., Müller, C., and Métraux, J. 1996b. Perception of free cutin monomers by plant cells. Plant J. 10:331-341.

Schweizer, P., Jeanguenat, A., Whitacre, D., Metraux, J. P., and Mosinger, E. 1996a. Induction of resistance in barley against Erysiphe graminis f. sp. hordei by free cutin monomers. Physiol. Mol. Plant Pathol. 49: 103-120.

Skamnioti, P., Furlong, R. F., and Gurr, S. J. 2008. Evolutionary history of the ancient cutinase family in five filamentous Ascomycetes reveals differential gene duplications and losses and in Magnaporthe grisea shows evidence of sub- and neo-functionalization. New Phytol. 180: 711-721.

Skamnioti, P., and Gurr, S. J. 2007. Magnaporthe grisea cutinase2 mediates appressorium differentiation and host penetration and is required for full virulence. Plant Cell 19:2674-2689.

Stahl, D. J., and Schäfer, W. 1992. Cutinase is not required for fungal pathogenicity on pea. Plant Cell 4:621-629.

Sweigard, J. A., Chumley, F. G., and Valent, B. 1992. Disruption of a Magnaporthe grisea cutinase gene. Mol. Gen. Genet. 232:183-190.

Tamura, K., Stecher, G., Peterson, D., Filipski, A., and Kumar, S. 2013. MEGA6: Molecular evolutionary genetics analysis version 6.0. Mol. Biol. Evol. 30:2725-2729.

Thomas, R., Fang, X., Ranathunge, K., Anderson, T. R., Peterson, C. A., and Bernards, M. A. 2007. Soybean root suberin: Anatomical distribution, chemical composition, and relationship to partial resistance to Phytophthora sojae. Plant Physiol. 144:299-311.

Thomma, B. P., Nürnberger, T., and Joosten, M. H. 2011. Of PAMPs and effectors: The blurred PTI-ETI dichotomy. Plant Cell 23:4-15.
Trail, F., and Koller, W. 1994. Diversity of cutinases from plant pathogenic fungi: Purification and characterization of two cutinases from Alternaria brassicicola. Physiol. Mol. Plant Pathol. 42:81-92.

van der Vlugt-Bergmans, C. J., Wagemakers, C. A., and van Kan, J. A. 1997. Cloning and expression of the cutinase A gene of Botrytis cinerea. Mol. Plant-Microbe Interact. 10:21-29.

Wang, Y., Chen, J., Li, D. W., Zheng, L., and Huang, J. 2017. CglCUT1 gene required for cutinase activity and pathogenicity of Colletotrichum gloeosporioides causing anthracnose of Camellia oleifera. Eur. J. Plant Pathol. 147:103-114.

Wu, Y., and Zhou, J. M. 2013. Receptor-like kinases in plant innate immunity. J. Integr. Plant Biol. 55:1271-1286.

Yamada, K., Yamashita-Yamada, M., Hirase, T., Fujiwara, T., Tsuda, K. Hiruma, K., and Saijo, Y. 2016. Danger peptide receptor signaling in plants ensures basal immunity upon pathogen-induced depletion of BAK1. EMBO J. 35:46-61.

Yamaguchi, Y., Huffaker, A., Bryan, A. C., Tax, F. E., and Ryan, C. A 2010. PEPR2 is a second receptor for the Pep1 and Pep2 peptides and contributes to defense responses in Arabidopsis. Plant Cell 22:508-522.

Yamaguchi, Y., Pearce, G., and Ryan, C. A. 2006. The cell surface leucinerich repeat receptor for AtPep1, an endogenous peptide elicitor in Arabidopsis, is functional in transgenic tobacco cells. Proc. Natl. Acad. Sci. U.S.A. 103:10104-10109.

Zhang, H., Wu, Q., Cao, S., Zhao, T., Chen, L., Zhuang, P., Zhou, X., and Gao, Z. 2014. A novel protein elicitor (SsCut) from Sclerotinia sclerotiorum induces multiple defense responses in plants. Plant Mol. Biol. 86:495-511.

Zhang, W., Fraiture, M., Kolb, D., Löffelhardt, B., Desaki, Y., Boutrot, F. F., Tör, M., Zipfel, C., Gust, A. A., and Brunner, F. 2013. Arabidopsis receptorlike protein 30 and receptor-like kinase suppressor of BIR1-1/EVERSHED mediate innate immunity to necrotrophic fungi. Plant Cell 25:4227-4241.

Zhang, Z., Henderson, C., Perfect, E., Carver, T. L., Thomas, B. J., Skamnioti, P., and Gurr, S. J. 2005. Of genes and genomes, needles and haystacks: Blumeria graminis and functionality. Mol. Plant Pathol. 6:561-575.

Zhao, Z., Liu, H., Wang, C., and Xu, J. R. 2014. Correction: Comparative analysis of fungal genomes reveals different plant cell wall-degrading capacity in fungi. BMC Genomics 15:6.

Zipfel, C. 2008. Pattern-recognition receptors in plant innate immunity. Curr. Opin. Immunol. 20:10-16.

Zipfel, C. 2014. Plant pattern-recognition receptors. Trends Immunol. 35:345-351. 